\title{
Annexing magic and tune-out wavelengths to the clock transitions of the alkaline-earth metal ions
}

\author{
Jasmeet Kaur*, Sukhjit Singh and Bindiya Arora ${ }^{\dagger}$ \\ Department of Physics, Guru Nanak Dev University, Amritsar, Punjab-143005, India \\ B. K. Sahoo \\ Atomic, Molecular and Optical Physics Division, \\ Physical Research Laboratory, Navrangpura, Ahmedabad-380009, India
}

(Dated: Received date; Accepted date)

\begin{abstract}
We present additional magic wavelengths $\left(\lambda_{\text {magic }}\right)$ for the clock transitions in the alkaline-earth metal ions considering circular polarized light aside from our previously reported values in [J. Kaur et al., Phys. Rev. A 92, 031402(R) (2015)] for the linearly polarized light. Contributions from the vector component to the dynamic dipole polarizabilities $\left(\alpha_{d}(\omega)\right)$ of the atomic states associated with the clock transitions play major roles in the evaluation of these $\lambda_{\text {magic }}$, hence facilitating in choosing circular polarization of lasers in the experiments. Moreover, the actual clock transitions in these ions are carried out among the hyperfine levels. The $\lambda_{\text {magic }}$ values in these hyperfine transitions are estimated and found to be different from $\lambda_{\text {magic }}$ for the atomic transitions due to different contributions coming from the vector and tensor part of $\alpha_{d}(\omega)$. Importantly, we also present $\lambda_{\text {magic }}$ values that depend only on the scalar component of $\alpha_{d}(\omega)$ for their uses in a specially designed trap geometry for these ions so that they can be used unambiguously among any hyperfine levels of the atomic states of the clock transitions. We also present $\alpha_{d}(\omega)$ values explicitly at the $1064 \mathrm{~nm}$ for the atomic states associated with the clock transitions which may be useful for creating "high-field seeking" traps for the above ions using the Nd:YAG laser. The tune out wavelengths at which the states would be free from the Stark shifts are also presented. Accurate values of the electric dipole matrix elements required for these studies are given and trends of electron correlation effects in determining them are also highlighted.
\end{abstract}

PACS numbers: 32.60.+i, 37.10.Jk, 32.10.Dk

\section{INTRODUCTION}

Atomic clocks based on optical lattices are capable of proffering outstanding stable and accurate time keeping devices. A fundamental feature of an optical lattice clock is that it interrogates an optical transition with the controlled atomic motion [1]. At present, the most stable clock is based on the optical lattices of ${ }^{87} \mathrm{Sr}$ atoms with accuracy below $10^{-18}$ [2]. The unique feature of this clock is that the atoms are trapped at the wavelengths of an external electric field at which the differential light shift of an atomic transition nullifies. These wavelengths are commonly known as the magic wavelengths ( $\left.\lambda_{\text {magic }}\right)$ [3]. However, ions provide more accurate atomic clocks since various systematics in the ions can be controlled better [4, 5]. As a result a number of ions, such as, ${ }^{27} \mathrm{Al}^{+}$ ${ }^{199} \mathrm{Hg}^{+},{ }^{171} \mathrm{Yb}^{+},{ }^{115} \mathrm{In}^{+},{ }^{88} \mathrm{Sr}^{+},{ }^{40} \mathrm{Ca}^{+},{ }^{113} \mathrm{Cd}^{+}$etc. are under consideration for building accurate clocks. Among the various ions proposed for frequency standards [4, 6 9], the alkaline earth metal ions possess an advantage that transitions required for cooling and re-pumping of ions and clock frequency measurement can be easily accessed using non-bulky solid state or diode lasers [4].

\footnotetext{
*Email: jasmeetphy.rsh@gndu.ac.in

†Email: bindiya.phy@gndu.ac.in

${ }_{\ddagger}$ Email: bijaya@prl.res.in
}

Moreover presence of metastable $D$ states in these ions, whose lifetimes range from milliseconds to several seconds, assist in carrying out measurements meticulously.

The recent development on measurement of $\lambda_{\text {magic }}$ in a singly charged ${ }^{40} \mathrm{Ca}^{+}$ion has now open-up a platform for the possibility of building up pure optical trapped ion clocks [10]. Optical lattices blended with unique features of optical transitions in an ionic system can revolutionize the secondary as well as primary frequency standards. However, the potential of the optical dipole trap perturb the energy levels of the ion unevenly and the consistency of an ion optical clock depreciates. Therefore, knowledge of $\lambda_{\text {magic }}$ values in these ions would be instrumental for constructing all-optical trapped ion clocks. These wavelengths can be found out using accurate values of the dynamic dipole polarizabilities $\alpha_{d}(\omega)$ of the states associated with the clock transitions. Also, information on the dynamic $\left(\alpha_{d}(\omega)\right)$ values, especially at which the ions are being trapped will be of great significance. Improved atomic clocks will obviously ease the widely used technologies including precise determination of fundamental constants 11], accurate control of quantum states [12] and advancement in communication, Global Positioning System [13] etc.

Following the measurement of $\lambda_{\text {magic }}$ in the $4 S_{1 / 2}-$ $3 D_{5 / 2}$ transition of ${ }^{43} \mathrm{Ca}^{+}$, we had investigated these values in the $n S_{1 / 2}-(n-1) D_{3 / 2}$ and $n S_{1 / 2}-(n-1) D_{5 / 2}$ transitions of the alkaline-earth ions [14] for the ground state principal quantum number $n$. However, those stud- 
ies were focused mainly on the linearly polarized light limiting the choice for experimental measurements. Application of circular polarized light to atomic systems introduce contributions from the vector polarizabilities in the Stark shifts of the energy levels, which is linearly proportional to the angular frequency of the applied field. This can help in manipulating the Stark shifts in the energy levels and can lend to more degrees of freedom to attain further $\lambda_{\text {magic }}$ values as per the experimental stipulation. Moreover, it is advantageous to consider hyperfine transitions in certain isotopes of the singly charged alkaline-earth ions, giving rise to zero hyperfine angular momentum, to get-rid off the systematics due to electric quadrupole shifts [15 17]. Since $\alpha_{d}$ values of atomic and hyperfine states in an atomic system are different when vector and tensor components of $\alpha_{d}$ contribute, the $\lambda_{\text {magic }}$ values also differ between the atomic and hyperfine transitions. Thus, it would be pertinent to investigate $\lambda_{\text {magic }}$ both in the atomic and hyperfine transitions before the experimental consideration of the proposed ions. In fact, it could be more convenient to have $\lambda_{\text {magic }}$ that are independent of choice of both magnetic and hyperfine sub-levels in a given clock transition.

In fact, the Nd:YAG lasers at $1064 \mathrm{~nm}$ is often used for trapping atoms and ions because of their relatively high power and low intensity noise [18]. Traps built with long wavelength lasers are generally high-field-seeking where the atoms are attracted to the intensity maxima. Dynamic polarizabilities for the considered ions at $1064 \mathrm{~nm}$ will be of immense interest to the experimentalists since these polarizabilities will be immediately useful for operating optical traps at $1064 \mathrm{~nm}$ light fields.

In the present work, we aim to search for the $\lambda_{\text {magic }}$ for the $n S_{1 / 2}-(n-1) D_{3 / 2,5 / 2}$ optical clock transitions, both in the atomic and hyperfine levels, in ${ }^{43} \mathrm{Ca}^{+}$(with nuclear spin $I=7 / 2),{ }^{87} \mathrm{Sr}^{+}(I=9 / 2)$ and ${ }^{137} \mathrm{Ba}^{+}(I=3 / 2)$ ions using circularly polarized light. These values can be compared with the values for the linearly polarized light reported in Ref. [14] for the experimental consideration to trap the above ions. Also, we had demonstrated in a recent work how a trap geometry can be chosen in such a way that Stark shifts observed by the energy levels can be free from the contributions from the vector and tensor components of the $\alpha_{d}$ of the atomic states [27]. Assuming such trapping geometries for the considered alkaline-earth ions, we also give $\lambda_{\text {magic }}$ values using only the scalar polarizability contributions. Moreover, we identify the tune-out wavelengths $\left(\lambda_{\mathrm{T}}\right)$ of the respective states for which the dynamic dipole polarizability of these ions vanishes. Comprehension of these $\lambda_{\mathrm{T}}$ values are needed for the sympathetic cooling of other possible singly and multiply charged ions in two-species mixtures with the considered alkaline earth ions [5, 8, 28]. We also present dynamic polarizability of these states at the $1064 \mathrm{~nm}$ wavelength of the applied external electric field. Contributions from various electric dipole (E1) matrix elements in determining the $\alpha_{d}$ values and the role of the electron correlation effects for the evaluation of accurate values of the E1 matrix elements are also discussed. Unless stated otherwise, all the results are given in atomic unit (a.u.) throughout this paper.

\section{THEORY}

The Stark shift in the energy of $K^{\text {th }}$ level of an atom placed in an electric field is given by [29, 30]

$$
\Delta E^{K}=-\frac{1}{4} \alpha_{d}^{K}(\omega) \mathcal{E}^{2},
$$

where $\mathcal{E}$ is the amplitude of the external electric field due to the applied laser in an atomic system and $\alpha_{d}^{K}(\omega)$ is the dynamic dipole polarizability for the state $K$ with its magnetic projection $M$. In tensor decomposition, $\alpha_{d}^{K}$ can be expressed as

$$
\begin{aligned}
\alpha_{d}^{K}(\omega)= & \alpha_{d, 0}^{K}(\omega)+\beta(\epsilon) \frac{M}{2 K} \alpha_{d, 1}^{K}(\omega) \\
& +\gamma(\epsilon) \frac{3 M^{2}-K(K+1)}{K(2 K-1)} \alpha_{d, 2}^{K}(\omega),
\end{aligned}
$$

where $\alpha_{d, i}^{K}(\omega)$ with $i=0,1,2$ are the scalar, vector and tensor components of $\alpha_{d}^{K}(\omega)$ respectively. In the specific cases, $K$ can be either the atomic angular momentum $J$ or hyperfine angular momentum $F$. The terms $\beta(\epsilon)$ and $\gamma(\epsilon)$ are defined as [31]

$$
\beta(\epsilon)=\iota\left(\hat{\epsilon} \times \hat{\epsilon}^{*}\right) \cdot \hat{e}_{B}
$$

and

$$
\gamma(\epsilon)=\frac{1}{2}\left[3\left(\hat{\epsilon}^{*} \cdot \hat{e}_{B}\right)\left(\hat{\epsilon} \cdot \hat{e}_{B}\right)-1\right],
$$

with the quantization axis unit vector $\hat{e}_{B}$ and polarization unit vector $\hat{e}$. The differential Stark shift of a transition between states $K$ to $K^{\prime}$ can be formulated as

$$
\begin{aligned}
\delta E_{K K^{\prime}}= & \Delta E_{K}-\Delta E_{K^{\prime}}=-\frac{1}{2}\left[\left\{\alpha_{d, 0}^{K}(\omega)-\alpha_{d, 0}^{K^{\prime}}(\omega)\right\}\right. \\
& +\beta(\epsilon)\left\{\frac{M_{K}}{2 K} \alpha_{d, 1}^{K}(\omega)-\frac{M_{K^{\prime}}}{2 K^{\prime}} \alpha_{d, 1}^{K^{\prime}}(\omega)\right\} \\
& +\gamma(\epsilon)\left\{\frac{3 M_{K}^{2}-K(K+1)}{K(2 K-1)} \alpha_{d, 2}^{K}(\omega)\right. \\
& \left.\left.-\frac{3 M_{K^{\prime}}^{2}-K^{\prime}\left(K^{\prime}+1\right)}{K^{\prime}\left(2 K^{\prime}-1\right)} \alpha_{d, 2}^{K^{\prime}}(\omega)\right\}\right] \mathcal{E}^{2}
\end{aligned}
$$

To obtain null differential Stark shift, it is obvious from Eq. (5) that either the independent components of the polarizabilities cancel out each other or the net resultant nullifies which depend upon the choice of $\beta(\epsilon), \gamma(\epsilon)$, $M_{K}$ and $M_{K^{\prime}}^{\prime}$ magnetic sublevels. Moreover, as we have demonstrated recently, the differential Stark shift can be independent of the vector and tensor components of the states involved in a transition for a certain trap geometry [27]. Such trapping scheme is useful for $M_{J}, F$ and 
TABLE I: Calculated values of the static dipole polarizabilities $\alpha_{d, i}^{J}(0)$ for $n S_{1 / 2}$ and $(n-1) D_{3 / 2,5 / 2}$ states of ${ }^{43} \mathrm{Ca}^{+},{ }^{87} \mathrm{Sr}^{+}$ and ${ }^{137} \mathrm{Ba}^{+}$alkaline earth metal ions. Polarizability values are compared with the other available theoretical and experimental results. References are given in square brackets. Uncertainties in the results are given in parentheses.

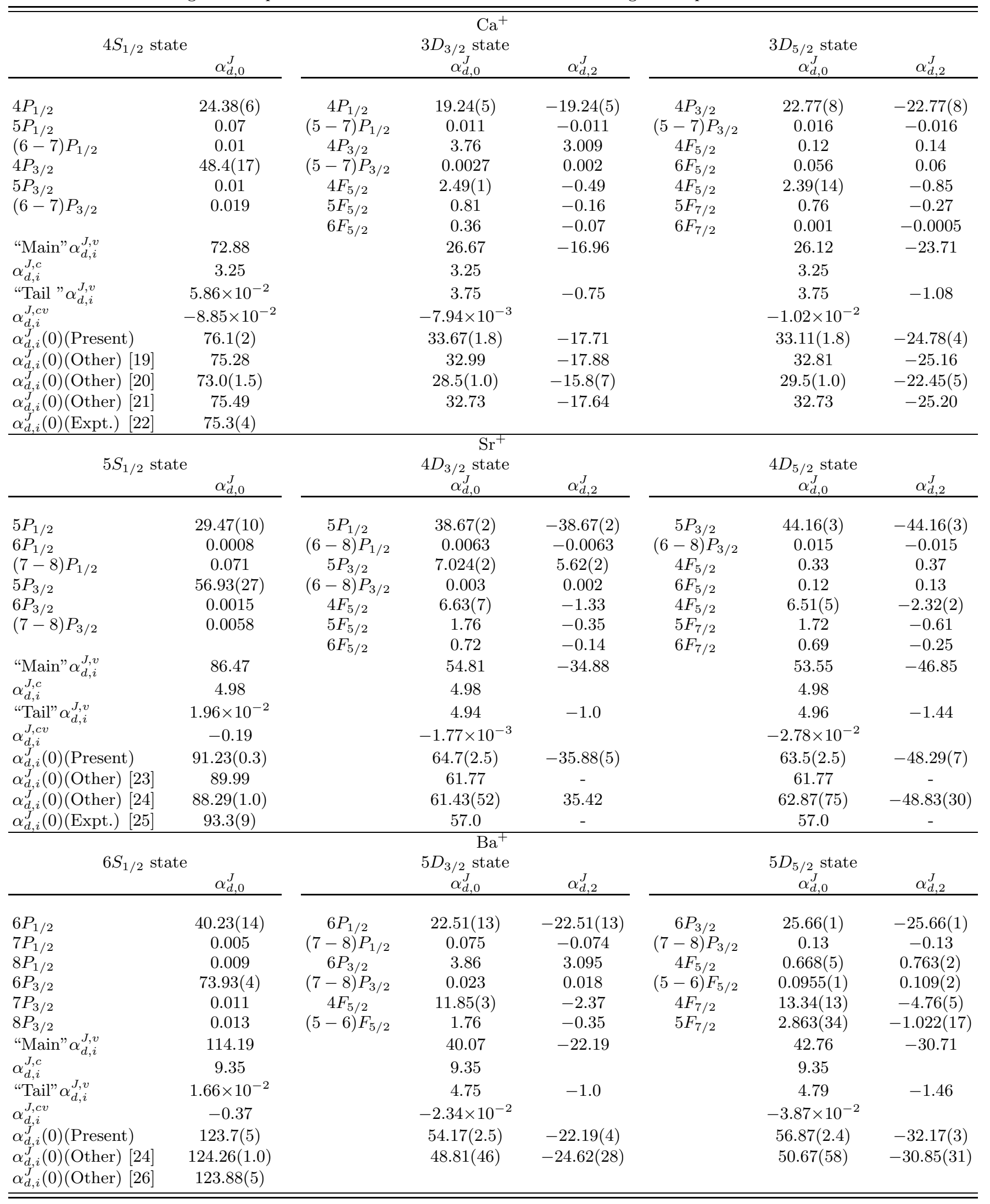


TABLE II: Calculated values of the dynamic dipole polarizabilities $\alpha_{d, i}^{J}(\omega)$ for the $n S_{1 / 2}$ and $(n-1) D_{3 / 2,5 / 2}$ states of ${ }^{43} \mathrm{Ca}^{+}$, ${ }^{87} \mathrm{Sr}^{+}$and ${ }^{137} \mathrm{Ba}^{+}$ions at $\lambda=1064 \mathrm{~nm}$ (equivalent to $\omega \simeq 0.035$ a.u.). Uncertainties in the results are given in parentheses.

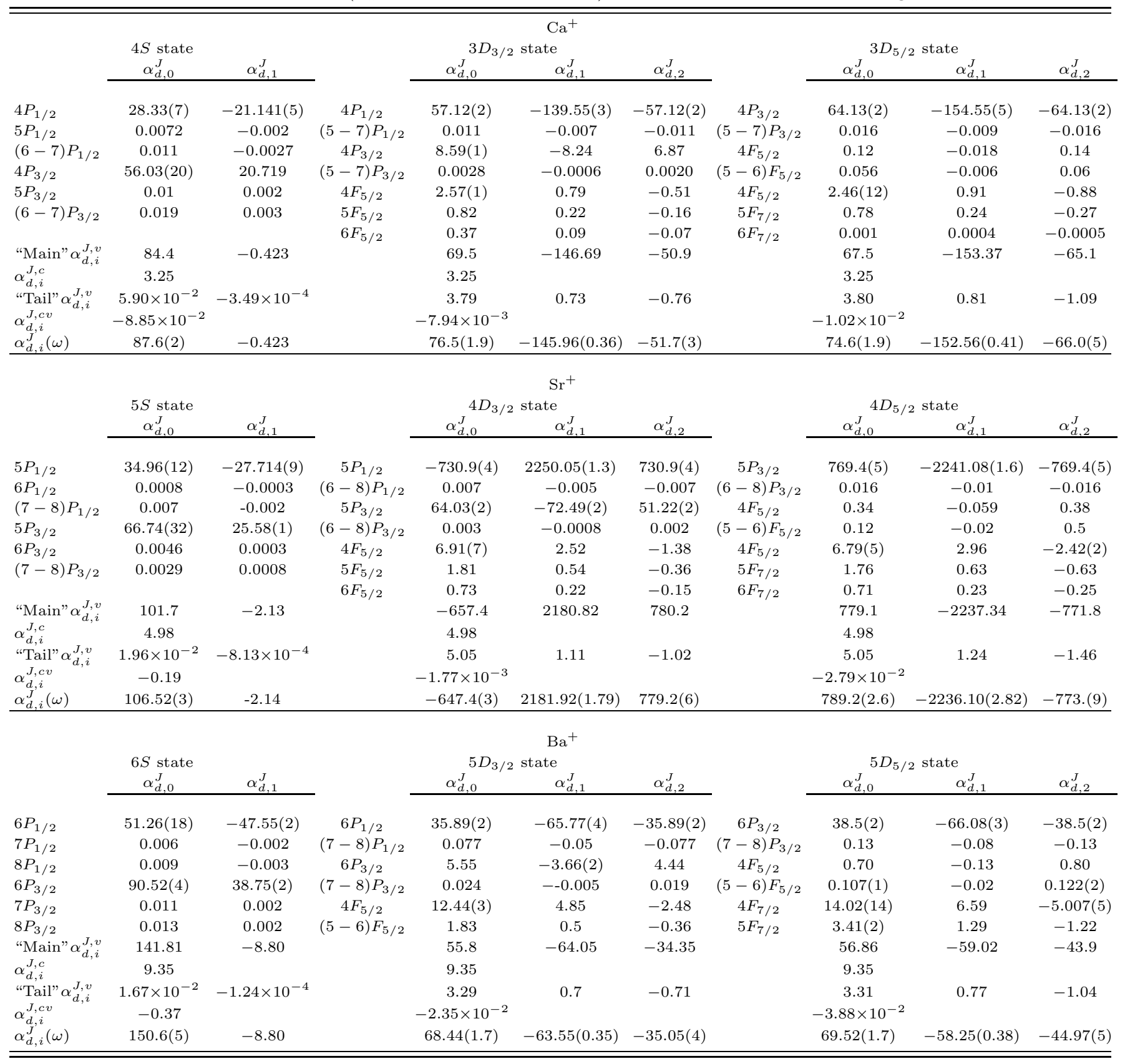


$M_{F}$ insensitive trapping and can be suitably applied for considered clock transitions in alkaline earth metal ions.

Conveniently the expressions for polarizabilities of the hyperfine and atomic levels can be expressed as 27]

$$
\begin{aligned}
\alpha_{d}^{F}(\omega) & =\alpha_{d, 0}^{F}(\omega)+\alpha_{d, 1}^{F}(\omega) \frac{A \cos \theta_{k} M_{F}}{2 F}+\alpha_{d, 2}^{F}(\omega) \\
& \times\left(\frac{3 \cos ^{2} \theta_{p}-1}{2}\right)\left[\frac{3 M_{F}^{2}-F(F+1)}{F(2 F-1)}\right],
\end{aligned}
$$

and

$$
\begin{aligned}
\alpha_{d}^{J}(\omega) & =\alpha_{d, 0}^{J}(\omega)+\alpha_{d, 1}^{J}(\omega) \frac{A \cos \theta_{k} M_{J}}{2 J}+\alpha_{d, 2}^{J}(\omega) \\
& \times\left(\frac{3 \cos ^{2} \theta_{p}-1}{2}\right)\left[\frac{3 M_{J}^{2}-J(J+1)}{J(2 J-1)}\right],
\end{aligned}
$$

where $\alpha_{d, i}^{K=J, F}(\omega)$ with $i=0,1,2$ are the scalar, vector and tensor components of the respective polarizabilities, $A$ represents degree of polarization, $\theta_{k}$ is the angle between the quantization axis and wave vector, and $\theta_{p}$ is the angle between the quantization axis and direction of polarization of the field. In the presence of magnetic field, $\cos \theta_{k}$ and $\cos ^{2} \theta_{p}$ can have any values depending on the direction of applied magnetic field. In the absence of magnetic field, $\cos \theta_{k}=0$ and $\cos ^{2} \theta_{p}=1$ for the linearly polarized light, where polarization vector is assumed to be along the quantization axis. However it yields $\cos \theta_{k}=1$ and $\cos ^{2} \theta_{p}=0$ for the circularly polarized light, where wave vector is assumed to be along the quantization axis. Polarizabilities of the hyperfine states can be evaluated from the atomic state results using the relations [31, 32]

$$
\begin{aligned}
\alpha_{d, 0}^{F}(\omega) & =\alpha_{d, 0}^{J}(\omega), \\
\alpha_{d, 1}^{F}(\omega) & =(-1)^{J+F+I+1}\left\{\begin{array}{ccc}
F & J & I \\
J & F & 1
\end{array}\right\} \\
& \times \sqrt{\frac{F(2 F+1)(2 J+1)(J+1)}{J(F+1)}} \alpha_{d, 1}^{J}(\omega)
\end{aligned}
$$

and

$$
\begin{aligned}
\alpha_{d, 2}^{F}(\omega) & =(-1)^{J+F+I}\left\{\begin{array}{lll}
F & J & I \\
J & F & 2
\end{array}\right\} \\
& \times \sqrt{\frac{F(2 F-1)(2 F+1)}{(2 F+3)(F+1)}} \\
& \times \sqrt{\frac{(2 J+3)(2 J+1)(J+1)}{J(2 J-1)}} \alpha_{d, 2}^{J}(\omega) .
\end{aligned}
$$

Further, we can evaluate the atomic dipole polarizabil- ities using the expressions

$$
\begin{aligned}
\alpha_{d, 0}^{J}(\omega)= & \frac{2}{3(2 J+1)} \sum_{J^{\prime}} \frac{\left(E-E^{\prime}\right)\left|\left\langle J|| \mathbf{D} \| J^{\prime}\right\rangle\right|^{2}}{\omega^{2}-\left(E-E^{\prime}\right)^{2}}, \\
\alpha_{d, 1}^{J}(\omega)= & \sqrt{\frac{24 J}{(J+1)(2 J+1)} \sum_{J^{\prime}}(-1)^{J+J^{\prime}}} \\
& \times\left\{\begin{array}{ccc}
J & 1 & J \\
1 & J^{\prime} & 1
\end{array}\right\} \frac{\omega\left|\left\langle J|| \mathbf{D} \| J^{\prime}\right\rangle\right|^{2}}{\omega^{2}-\left(E-E^{\prime}\right)^{2}}
\end{aligned}
$$

and

$$
\begin{aligned}
\alpha_{d, 2}^{J}(\omega) & =\sqrt{\frac{40 J(2 J-1)}{3(J+1)(2 J+3)(2 J+1)}} \sum_{J^{\prime}}(-1)^{J+J^{\prime}} \\
& \times\left\{\begin{array}{ccc}
J & 2 & J \\
1 & J^{\prime} & 1
\end{array}\right\} \frac{\left(E-E^{\prime}\right)\left|\left\langleJ \left\|\left.\left|\mathbf{D} \| J^{\prime}\right\rangle\right|^{2}\right.\right.\right.}{\omega^{2}-\left(E-E^{\prime}\right)^{2}}
\end{aligned}
$$

where $J^{\prime}$ s are the angular momentum of the intermediate states, $E$ and $E^{\prime}$ are energies of the corresponding states, $\left\langle J|| \mathbf{D} \| J^{\prime}\right\rangle$ are the reduced E1 matrix elements.

We define the tune-out wavelength $\lambda_{\mathrm{T}}$ as the wavelength where the dynamic polarizability of the state is zero. We have determined the tune out wavelengths for the ground, $(n-1) D_{3 / 2}$ and $(n-1) D_{5 / 2}$ states of ${ }^{43} \mathrm{Ca}^{+}$, ${ }^{87} \mathrm{Sr}^{+}$and ${ }^{137} \mathrm{Ba}^{+}$ions. The detailed description about these calculations have been given in the Refs. [33, 34].

\section{METHOD OF EVALUATION}

As discussed in our earlier works [14, 35, 36], each component of $\alpha_{d}^{J}(\omega)$ can be conveniently evaluated in the considered alkaline earth ions, in which many of the lowlying states have electronic configurations as a common closed core of inert gas atoms and a well defined valence orbital, by classifying into three different contributions such as

$$
\alpha_{d, i}^{J}(\omega)=\alpha_{d, i}^{J, c}(\omega)+\alpha_{d, i}^{J, c v}(\omega)+\alpha_{d, i}^{J, v}(\omega),
$$

where $\alpha_{d, i}^{J, c}, \alpha_{d, i}^{J, c v}$ and $\alpha_{d, i}^{J, v}$ are referred to as the core, core-valence and valence electron correlation contributions, respectively. Since the valence electron correlation effects are mainly estimated by $\alpha_{d, i}^{J, v}$, this gives majority contribution followed by $\alpha_{d, i}^{J, c}$. Again, accuracies in the $a b$ initio results of these quantities mainly suffer due to the uncertainties associated with the calculated energies of the atomic states. Therefore, we calculate only the E1 matrix elements of as many as low-lying states of the considered ions employing a relativistic coupledcluster (RCC) method, which is an all order perturbative method, and combine them with the experimental energy values from the National Institute of Science and Technology (NIST) database [37] to determine the dominant contributions to $\alpha_{d, i}^{J, v}$. We refer this as "Main" contribution to $\alpha_{d, i}^{J, v}$; while the smaller contributions coming from 
TABLE III: The $\lambda_{\text {magic }}$ values (in nm) with their corresponding polarizabilities $\alpha_{d}^{J}\left(\lambda_{\text {magic }}\right)$ (in a.u.) for the $n S_{1 / 2}\left(M_{J}=1 / 2\right) \rightarrow$ $(n-1) D_{3 / 2}$ transitions using circularly polarized light in the ${ }^{43} \mathrm{Ca}^{+},{ }^{87} \mathrm{Sr}^{+}$and ${ }^{137} \mathrm{Ba}^{+}$ions. The resonant wavelengths $\lambda_{\text {res }}$ to indicate the placement of the $\lambda_{\text {magic }}$ in between resonant transitions are also listed.

\begin{tabular}{|c|c|c|c|c|c|c|c|c|c|}
\hline \multirow[b]{2}{*}{ Resonances } & \multirow[b]{2}{*}{$\lambda_{\text {res }}$} & \multicolumn{2}{|c|}{$\overline{M_{J}=3 / 2}$} & \multicolumn{2}{|c|}{$\overline{M_{J}=1 / 2}$} & \multicolumn{2}{|c|}{$\overline{M_{J}=-1 / 2}$} & \multicolumn{2}{|c|}{$\overline{M_{J}=-3 / 2}$} \\
\hline & & $\lambda_{\text {magic }}$ & $\alpha_{d}^{J}\left(\lambda_{\text {magic }}\right)$ & $\lambda_{\text {magic }}$ & $\alpha_{d}^{J}\left(\lambda_{\text {magic }}\right)$ & $\lambda_{\text {magic }}$ & $\alpha_{d}^{J}\left(\lambda_{\text {magic }}\right)$ & $\lambda_{\text {magic }}$ & $\alpha_{d}^{J}\left(\lambda_{\text {magic }}\right)$ \\
\hline${ }^{43} \mathrm{Ca}^{+}$ & $4 S_{1 / 2}-3 D_{3 / 2}$ & & & & & & & & \\
\hline $4 S_{1 / 2}-4 P_{3 / 2}$ & 393.366 & $2046(4)$ & 2034 & $2046(0)$ & 282 & $2046(10)$ & & & \\
\hline $\begin{array}{l}4 S_{1 / 2}-4 P_{1 / 2} \\
3 D_{3 / 2}-4 P_{3 / 2}\end{array}$ & $\begin{array}{l}396.847 \\
849.802\end{array}$ & $394.6(4)$ & -20.34 & $394.6(9)$ & -2.82 & $394.6(10)$ & 11.68 & $394.6(10)$ & 22.40 \\
\hline $3 D_{3 / 2}-4 P_{1 / 2}$ & 866.214 & $850.9(2)$ & 96.33 & $853.1(2)$ & 96.20 & - & - & - & - \\
\hline${ }^{87} \mathrm{Sr}^{+}$ & $5 S_{1 / 2}-4 D_{3 / 2}$ & $1467.8(4)$ & 81.31 & $1013.4(5)$ & 89.31 & $870.7(3)$ & 95.15 & - & - \\
\hline $5 S_{1 / 2}-5 P_{3 / 2}$ & 407.7714 & $412.5(9)$ & -20.62 & $412.4(9)$ & 3.99 & $412.4(9)$ & 27.18 & $412.3(8)$ & 48.55 \\
\hline $\begin{array}{l}5 S_{1 / 2}-5 P_{1 / 2} \\
4 D_{3 / 2}-5 P_{3 / 2}\end{array}$ & $\begin{array}{c}421.5524 \\
1003.6654\end{array}$ & & & & & & & & \\
\hline $4 D_{3 / 2}-5 P_{1 / 2}$ & 1091.4874 & $1009.3(2)$ & 109.74 & $1019.7(3)$ & 109.29 & $1062.5(3)$ & 107.65 & - & - \\
\hline${ }^{137} \mathrm{Ba}^{+}$ & $6 S_{1 / 2}-5 D_{3 / 2}$ & - & - & $1577.2(3)$ & 98.17 & - & - & - & - \\
\hline $\begin{array}{l}6 S_{1 / 2}-6 P_{3 / 2} \\
6 S_{1 / 2}-6 P_{1 / 2} \\
5 D_{3 / 2}-6 P_{3 / 2}\end{array}$ & $\begin{array}{l}455.4033 \\
493.4077 \\
585.3675\end{array}$ & $468.8(4)$ & -70.91 & $468.1(4)$ & -13.13 & $467.6(5)$ & 30.14 & $467.3(4)$ & 59.27 \\
\hline $5 D_{3 / 2}-6 P_{1 / 2}$ & 649.6898 & $\begin{array}{l}587.6(9) \\
841.7(5)\end{array}$ & 373.89 & $589.5(3)$ & 368.60 & $589.6(5)$ & 368.35 & - & - \\
\hline
\end{tabular}

the high-lying excited states are estimated in the ab initio formalism using the Dirac-Hartree-Fock (DHF) method and are mentioned as "Tail" contribution to $\alpha_{d, i}^{J, v}$. To estimate the other two contributions, it is not possible to use sum-over-states approach, so we determine $\alpha_{d, i}^{J, c}$ in the random-phase approximation (RPA). This included core-polarization effects to all orders. It has been found that RPA can give these values reliably for the inert gas configured atomic systems [38]. Again as demonstrated later, the $\alpha_{d, i}^{J, c v}$ contributions are very small in these ions. Thus, we evaluate them using the DHF method.

A small number of E1 matrix elements of the $n D-n F$ transitions of ${ }^{137} \mathrm{Ba}^{+}$are borrowed from the work of Sahoo et al. [39], while the rest E1 matrix elements for the evaluation of "Main" contribution to $\alpha_{d}^{J, v}$ are obtained considering the singles and doubles excitation approximation in the RCC (SD) method as described in Refs. [40, 41]. In the SD method, the wave function of the state with the closed-core with a valence electron $v$ is represented as an expansion

$$
\begin{aligned}
\left|\Psi_{v}\right\rangle_{\mathrm{SD}}= & {\left[1+\sum_{m a} \rho_{m a} a_{m}^{\dagger} a_{a}+\frac{1}{2} \sum_{m n a b} \rho_{m n a b} a_{m}^{\dagger} a_{n}^{\dagger} a_{b} a_{a}\right.} \\
& \left.+\sum_{m \neq v} \rho_{m v} a_{m}^{\dagger} a_{v}+\sum_{m n a} \rho_{m n v a} a_{m}^{\dagger} a_{n}^{\dagger} a_{a} a_{v}\right]\left|\Phi_{v}\right\rangle
\end{aligned}
$$

where $\left|\Phi_{v}\right\rangle$ is the DHF wave function of the state. In the above expression, $a_{i}^{\dagger}$ and $a_{i}$ are the creation and annihilation operators with the indices $\{m, n\}$ and $\{a, b\}$ designating the virtual and core orbitals of $\left|\Phi_{v}\right\rangle, \rho_{m a}$ and $\rho_{m v}$ are the corresponding single core and valence excitation coefficients, and $\rho_{m n a b}$ and $\rho_{m n v a}$ are the double core and valence excitation coefficients. To obtain the DHF wave function, we use a finite size basis set consisting of 70 B-splines constrained to a large cavity of radius $\mathrm{R}=$ 220 a.u. and solved self consistently using the Roothan equation on a nonlinear grid.

In order to verify contributions from the higher level excitations, we also estimate leading order contributions from the triple excitations perturbatively in the SD method framework (SDpT method) by expressing the 
TABLE IV: The $\lambda_{\text {magic }}$ values (in $\mathrm{nm}$ ) with their corresponding polarizabilities $\alpha_{d}^{J}\left(\lambda_{\text {magic }}\right)$ (in a.u.) for the $n S_{1 / 2}\left(M_{J}=\right.$ $-1 / 2) \rightarrow(n-1) D_{3 / 2}$ transitions using circularly polarized light in the ${ }^{43} \mathrm{Ca}^{+},{ }^{87} \mathrm{Sr}^{+}$and ${ }^{137} \mathrm{Ba}^{+}$ions. The resonant wavelengths $\lambda_{\text {res }}$ to indicate the placement of the $\lambda_{\text {magic }}$ in between resonant transitions are also listed.

\begin{tabular}{|c|c|c|c|c|c|c|c|c|c|}
\hline \multirow[b]{2}{*}{ Resonances } & \multirow[b]{2}{*}{$\lambda_{\text {res }}$} & \multicolumn{2}{|c|}{$M_{J}=3 / 2$} & \multicolumn{2}{|c|}{$M_{J}=1 / 2$} & \multicolumn{2}{|c|}{$M_{J}=-1 / 2$} & \multicolumn{2}{|c|}{$M_{J}=-3 / 2$} \\
\hline & & $\lambda_{\text {magic }}$ & $\alpha_{d}^{j}\left(\lambda_{\text {magic }}\right)$ & $\lambda_{\text {magic }}$ & $\alpha_{d}^{j}\left(\lambda_{\text {magic }}\right)$ & $\lambda_{\text {magic }}$ & $\alpha_{d}^{J}\left(\lambda_{\text {magic }}\right)$ & $\lambda_{\text {magic }}$ & $\alpha_{d}^{J}\left(\lambda_{\text {magic }}\right)$ \\
\hline${ }^{43} \mathrm{Ca}^{+}$ & $4 S_{1 / 2}-3 D_{3 / 2}$ & & & & & & & & \\
\hline $3 D_{3 / 2}-4 P_{3 / 2}$ & 849.802 & & & & & & & & \\
\hline $3 D_{3 / 2}-4 P_{1 / 2}$ & 866.214 & $851.2(3)$ & 95.63 & $853.5(4)$ & 95.49 & - & - & - & - \\
\hline${ }^{87} \mathrm{Sr}^{+}$ & $5 S_{1 / 2}-4 D_{3 / 2}$ & $1549.9(5)$ & 80.95 & $1031.4(4)$ & 88.28 & $875.56(2)$ & 94.27 & - & - \\
\hline $5 S_{1 / 2}-5 P_{3 / 2}$ & 407.7714 & & & & & & & & \\
\hline $5 S_{1 / 2}-5 P_{1 / 2}$ & 421.5524 & $416.9(7)$ & -21.20 & $416.8(5)$ & 3.93 & $416.8(8)$ & 27.12 & $416.7(8)$ & 48.44 \\
\hline & & $\begin{array}{l}442.5(5) \\
478.2(5)\end{array}$ & $\begin{array}{l}-25.36 \\
-31.49\end{array}$ & $\begin{array}{l}442.3(6) \\
479.2(4)\end{array}$ & $\begin{array}{c}1.94 \\
-0.96\end{array}$ & $\begin{array}{l}442.1(2) \\
480.1(2)\end{array}$ & $\begin{array}{l}26.46 \\
25.56\end{array}$ & $\begin{array}{l}441.9(4) \\
481.1(1)\end{array}$ & $\begin{array}{l}48.03 \\
47.83\end{array}$ \\
\hline $4 D_{3 / 2}-5 P_{3 / 2}$ & 1003.6654 & $1009.2(1)$ & 106.69 & $1019.8(6)$ & 106.29 & $1064.8(5)$ & 104.79 & - & - \\
\hline $\begin{array}{l}4 D_{3 / 2}-5 P_{1 / 2} \\
{ }^{137} \mathrm{Ba}^{+}\end{array}$ & $\begin{array}{c}1091.4874 \\
6 S_{1 / 2}-5 D_{3 / 2}\end{array}$ & & - & - 1593.1(5) & 96.54 & - & - & - & - \\
\hline $6 S_{1 / 2}-6 P_{3 / 2}$ & 455.4033 & $479.2(5)$ & -79.42 & $478.5(4)$ & -17.21 & $477.9(1)$ & 28.76 & $477.5(2)$ & 58.89 \\
\hline $6 S_{1 / 2}-6 P_{1 / 2}$ & 493.4077 & $\begin{array}{l}505.4(1) \\
571.4(4)\end{array}$ & $\begin{array}{l}-106.86 \\
-314.04\end{array}$ & $\begin{array}{l}505.1(4) \\
574.1(2)\end{array}$ & $\begin{array}{c}-30.87 \\
-213.32\end{array}$ & $\begin{array}{l}504.8(2) \\
577.3(4)\end{array}$ & $\begin{array}{c}24.05 \\
-125.29\end{array}$ & $\begin{array}{c}504.7(2) \\
-\end{array}$ & $\begin{array}{c}57.93 \\
-\end{array}$ \\
\hline $\begin{array}{l}5 D_{3 / 2}-6 P_{3 / 2} \\
5 D_{3 / 2}-6 P_{1 / 2}\end{array}$ & 585.3675 & $589.4(4)$ & 47.82 & $595.5(2)$ & 89.44 & $602.4(2)$ & 120.417 & $590.5(2)$ & 56.46 \\
\hline & & $899.9(5)$ & 153.13 & $710.5(4)$ & 176.48 & - & - & - & - \\
\hline
\end{tabular}

TABLE V: The $\lambda_{\text {magic }}$ values (in nm) with their corresponding polarizabilities $\alpha_{d}^{J}\left(\lambda_{\text {magic }}\right)$ (in a.u.) for the $n S_{1 / 2}\left(M_{J}=1 / 2\right) \rightarrow$ $(n-1) D_{5 / 2}$ transitions using circularly polarized light in the ${ }^{43} \mathrm{Ca}^{+},{ }^{87} \mathrm{Sr}^{+}$and ${ }^{137} \mathrm{Ba}^{+}$ions. The resonant wavelengths $\lambda_{\text {res }}$ to indicate the placement of the $\lambda_{\text {magic }}$ in between resonant transitions are also listed.

\begin{tabular}{|c|c|c|c|c|c|c|c|c|c|c|c|c|c|}
\hline \multirow[b]{2}{*}{ Resonances } & \multirow[b]{2}{*}{$\lambda_{\text {res }}$} & \multicolumn{2}{|c|}{$M_{J}=5 / 2$} & \multicolumn{2}{|c|}{$M_{J}=3 / 2$} & \multicolumn{2}{|c|}{$M_{J}=1 / 2$} & \multicolumn{2}{|c|}{$M_{J}=-1 / 2$} & \multicolumn{2}{|c|}{$M_{J}=-3 / 2$} & \multicolumn{2}{|c|}{$M_{J}=-5 / 2$} \\
\hline & & $\lambda_{\text {magic }}$ & $\alpha_{d}^{J}\left(\lambda_{\text {magic }}\right)$ & $\lambda_{\text {magic }}$ & $\alpha_{d}^{J}\left(\lambda_{\text {magic }}\right)$ & $\lambda_{\text {magic }}$ & $\alpha_{d}^{J}\left(\lambda_{\text {magic }}\right)$ & $\lambda_{\text {magic }}$ & $\alpha_{d}^{J}\left(\lambda_{\text {magic }}\right)$ & $\lambda_{\text {magic }}$ & $\alpha_{d}^{J}\left(\lambda_{\text {magic }}\right)$ & $\lambda_{\text {magic }}$ & $\alpha_{d}^{J}\left(\lambda_{\text {magic }}\right)$ \\
\hline${ }^{43} \mathrm{Ca}^{+}$ & $4 S_{1 / 2}-3 D_{5 / 2}$ & & & & & & & & & & & & \\
\hline $4 S_{1 / 2}-4 P_{3 / 2}$ & 393.366 & & & & & & & & & & & & \\
\hline & & $394.64(2)$ & -25.67 & $394.64(3)$ & -9.69 & $394.64(2)$ & 1.0 & $394.63(4)$ & 6.33 & $394.63(3)$ & 17.0 & $394.63(3)$ & 22.39 \\
\hline $4 S_{1 / 2}-4 P_{1 / 2}$ & 396.847 & & & & & & & & & & & & \\
\hline $3 D_{5 / 2}-4 P_{3 / 2}$ & 854.209 & & & & & & & & & & & & \\
\hline & & - & - & $1150.39(2)$ & 85.95 & $975.6(4)$ & 90.54 & $891.4(3)$ & 94.09 & - & - & - & - \\
\hline${ }^{87} \mathrm{Sr}^{+}$ & $5 S_{1 / 2}-4 D_{5 / 2}$ & & & & & & & & & & & & \\
\hline $5 S_{1 / 2}-5 P_{3 / 2}$ & 407.7714 & & & & & & & & & & & & \\
\hline & & $412.5(3)$ & -26.94 & $412.46(2)$ & -10.05 & $412.42(4)$ & 6.98 & $412.38(4)$ & 24.16 & $412.35(3)$ & 37.15 & $412.31(3)$ & 54.61 \\
\hline $5 S_{1 / 2}-5 P_{1 / 2}$ & 421.5524 & & & & & & & & & & & & \\
\hline $4 D_{5 / 2}-5 P_{3 / 2}$ & 1032.7309 & & & & & & & & & & & & \\
\hline & & - & - & - & - & $1379.4(3)$ & 100.44 & $1130.8(4)$ & 105.46 & - & - & - & - \\
\hline${ }^{137} \mathrm{Ba}^{+}$ & $6 S_{1 / 2}-5 D_{5 / 2}$ & & & & & & & & & & & & \\
\hline $6 S_{1 / 2}-6 P_{3 / 2}$ & 455.4033 & & & & & & & & & & & & \\
\hline & & $469.1(5)$ & -95.43 & $468.5(1)$ & -45.31 & $467.9(4)$ & -3.93 & $467.6(4)$ & 29.01 & $467.3(1)$ & 53.75 & $467.1(1)$ & 70.27 \\
\hline $6 S_{1 / 2}-6 P_{1 / 2}$ & 493.4077 & & & & & & & & & & & & \\
\hline $5 D_{5 / 2}-6 P_{3 / 2}$ & 614.1713 & & & & & & & & & & & & \\
\hline & & $810.1(8)$ & 189.71 & $691(2)$ & 237.75 & $644.7(5)$ & 275.99 & $623.3(5)$ & 302.79 & - & - & - & - \\
\hline
\end{tabular}

atomic wave functions as [40, 42]

where $\rho_{m n r v a b}^{\text {pert }}$ is the perturbed triple valence excitation

$\left|\Psi_{v}\right\rangle_{\mathrm{SDpT}}=\left|\Psi_{v}\right\rangle_{\mathrm{SD}}+\frac{1}{6} \sum_{m n r a b} \rho_{m n r v a b}^{\text {pert }} a_{m}^{\dagger} a_{n}^{\dagger} a_{r}^{\dagger} a_{b} a_{a} a_{v}\left|\Phi_{v}\right\rangle$ 
TABLE VI: The $\lambda_{\text {magic }}$ values (in $\mathrm{nm}$ ) with their corresponding polarizabilities $\alpha_{d}^{J}\left(\lambda_{\text {magic }}\right)$ (in a.u.) for the $n S_{1 / 2}\left(M_{J}=\right.$ $-1 / 2) \rightarrow(n-1) D_{5 / 2}$ transitions using circularly polarized light in the ${ }^{43} \mathrm{Ca}^{+},{ }^{87} \mathrm{Sr}^{+}$and ${ }^{137} \mathrm{Ba}^{+}$ions. The resonant wavelengths $\lambda_{\text {res }}$ to indicate the placement of the $\lambda_{\text {magic }}$ in between resonant transitions are also listed.

\begin{tabular}{|c|c|c|c|c|c|c|c|c|c|c|c|c|c|}
\hline \multirow[b]{2}{*}{ Resonances } & \multirow[b]{2}{*}{$\lambda_{\text {res }}$} & \multicolumn{2}{|c|}{$M_{J}=5 / 2$} & \multicolumn{2}{|c|}{$M_{J}=3 / 2$} & \multicolumn{2}{|c|}{$M_{J}=1 / 2$} & \multicolumn{2}{|c|}{$M_{J}=-1 / 2$} & \multicolumn{2}{|c|}{$M_{J}=-3 / 2$} & \multicolumn{2}{|c|}{$M_{J}=-5 / 2$} \\
\hline & & $\lambda_{\text {magic }}$ & $\alpha_{d}^{J}\left(\lambda_{\text {magic }}\right)$ & $\lambda_{\text {magic }}$ & $\alpha_{d}^{J}\left(\lambda_{\text {magic }}\right)$ & $\lambda_{\text {magic }}$ & $\alpha_{d}^{J}\left(\lambda_{\text {magic }}\right)$ & $\lambda_{\text {magic }}$ & $\alpha_{d}^{J}\left(\lambda_{\text {magic }}\right)$ & $\lambda_{\text {magic }}$ & $\alpha_{d}^{J}\left(\lambda_{\text {magic }}\right)$ & $\lambda_{\text {magic }}$ & $\alpha_{d}^{J}\left(\lambda_{\text {magic }}\right)$ \\
\hline${ }^{43} \mathrm{Ca}^{+}$ & $4 S_{1 / 2}-3 D_{5 / 2}$ & & & & & & & & & & & & \\
\hline $4 S_{1 / 2}-4 P_{1 / 2}$ & 396.847 & & & & & & & & & & & & \\
\hline $3 D_{5 / 2}-4 P_{3 / 2}$ & 854.209 & & & & & & & & & & & & \\
\hline${ }^{87} \mathrm{Sr}^{+}$ & $5 S_{1 / 2}-4 D_{5 / 2}$ & - & - & $1173.5(2)$ & 85.12 & $982.7(2)$ & 89.77 & $893.4(3)$ & 93.37 & - & - & - & - \\
\hline $5 S_{1 / 2}-5 P_{3 / 2}$ & 407.7714 & & & & & & & & & & & & \\
\hline $5 S_{1 / 2}-5 P_{1 / 2}$ & 421.5524 & $416.93(4)$ & -27.93 & $416.88(3)$ & -9.02 & $416.85(4)$ & 7.66 & $416.81(4)$ & 23.82 & $416.77(1)$ & 38.63 & $416.74(3)$ & 52.53 \\
\hline & & $442.49(4)$ & -31.93 & $442.36(3)$ & -12.11 & $442.25(3)$ & 6.12 & $442.13(4)$ & 22.84 & $442.03(8)$ & 38.18 & $441.93(3)$ & 52.07 \\
\hline & & $477.99(5)$ & 39.14 & $478.85(1)$ & -16.77 & $479.32(7)$ & 3.55 & $479.98(9)$ & 21.77 & $480.65(1)$ & 37.83 & $481.28(7)$ & 51.73 \\
\hline $4 D_{5 / 2}-5 P_{3 / 2}$ & 1032.7309 & & & & & & & & & & & & \\
\hline${ }^{137} \mathrm{Ba}^{+}$ & $\underline{6 S_{1 / 2}-5 D_{5 / 2}}$ & - & - & - & - & $1391.4(3)$ & 98.46 & $1134.5(4)$ & 102.92 & - & - & - & - \\
\hline $6 S_{1 / 2}-6 P_{3 / 2}$ & 4455.4033 & $479.5(2)$ & -108.02 & $478.9(2)$ & -52.89 & $478.4(2)$ & -7.78 & $477.9(4)$ & 27.52 & $477.6(4)$ & 53.33 & $477.4(7)$ & 69.73 \\
\hline $6 S_{1 / 2}-6 P_{1 / 2}$ & 493.4077 & $505.5(2)$ & -149.78 & $505.3(4)$ & -78.36 & $505.04(41)$ & -20.96 & $504.8(4)$ & 25.56 & $504.76(4)$ & 52.23 & $504.69(7)$ & 68.53 \\
\hline & & $568.8(2)$ & -451.72 & $572.14(2)$ & -284.62 & $576.52(2)$ & -144.27 & $582.4(2)$ & -31.07 & $589.7(4)$ & 50.45 & $591.7(2)$ & 65.99 \\
\hline $5 D_{5 / 2}-6 P_{3 / 2}$ & 614.1713 & $894.3(8)$ & 153.62 & $729.9(2)$ & 173.67 & $665.9(3)$ & 180.26 & $632.6(5)$ & 171.84 & - & - & - & - \\
\hline
\end{tabular}

amplitudes.

After obtaining wave functions employing the SD and SDpT method, we determine the E1 matrix element for a given transition between the states $\left|\Psi_{v}\right\rangle$ and $\left|\Psi_{w}\right\rangle$ by evaluating the expression

$$
Z_{v w}=\frac{\left\langle\Psi_{v}|Z| \Psi_{w}\right\rangle}{\sqrt{\left\langle\Psi_{v} \mid \Psi_{v}\right\rangle\left\langle\Psi_{w} \mid \Psi_{w}\right\rangle}} .
$$

To find out the uncertainties with the calculated E1 matrix elements, we have carried out semi-empirical scaling of the wave functions that accounts for evaluation of missing correlations contributions to the wave functions from the approximated SD and SDpT methods. This procedure involves scaling of the excitation coefficients and reevaluation of the E1 matrix elements. The scaling factors are decided from the correlation energy trends in the SD and SDpT methods. Details regarding this scaling procedure are given in Ref. [43].

\section{RESULTS AND DISCUSSION}

Since valence correlation contributions are vital for accurate estimate of polarizabilities, we include the E1 matrix elements among the low-lying states up to $4 S-7 P$, $3 D-7 P$ and $3 D-6 F$ transitions in ${ }^{43} \mathrm{Ca}^{+}, 5 S-8 P$, $4 D-8 P$ and $4 D-6 F$ transitions in ${ }^{87} \mathrm{Sr}^{+}$and $6 S-8 P$, $5 D-8 P$ and $5 D-6 F$ transitions in ${ }^{137} \mathrm{Ba}^{+}$for the evaluation of the "Main" contributions. All these matrix elements are calculated using the RCC method described

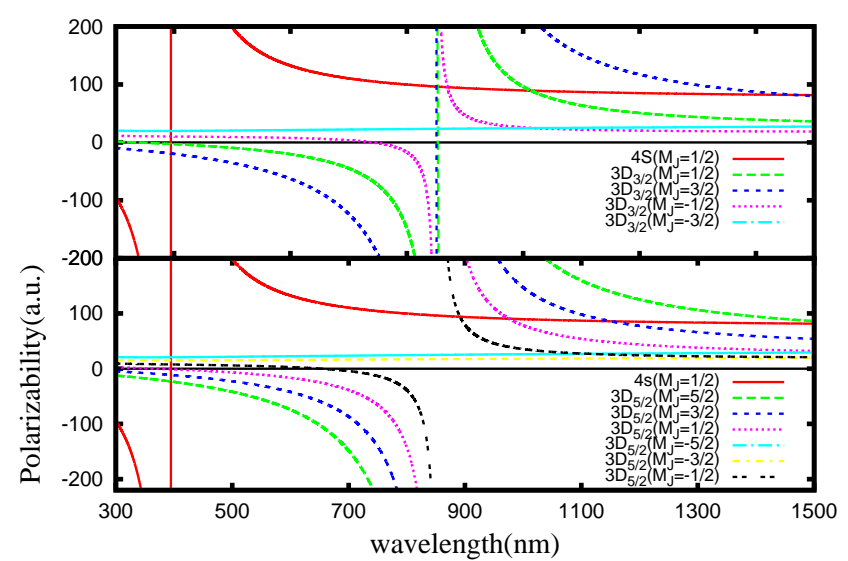

FIG. 1: (Color online) Dynamic dipole polarizabilities (in a.u.) for the $4 S_{1 / 2}$ and $3 D_{3 / 2,5 / 2}$ states of ${ }^{43} \mathrm{Ca}^{+}$with $A=-1$.

in the previous section. A few E1 matrix elements of the $n D-n F$ transitions of ${ }^{137} \mathrm{Ba}^{+}$are used from Ref. [39]. We present these considered E1 matrix elements in the Supplemental Material from different levels of approximation in the considered many-body methods. Scaled values to the SD and SDpT results, which mainly ameliorates the results by accounting corrections beyond the Brueckner-orbital contributions [40], are also given in the same table. We then give the "Final" results considering the most reliable values along with their estimated 
TABLE VII: The $\lambda_{\text {magic }}$ values (in $\mathrm{nm}$ ) and their corresponding polarizabilities $\alpha_{d}^{F}\left(\lambda_{\text {magic }}\right)$ (in a.u.) for the $n S_{1 / 2}\left|F, M_{F}\right\rangle \rightarrow$ $(n-1) D_{3 / 2,5 / 2}\left|F^{\prime}, M_{F^{\prime}}\right\rangle$ transitions in ${ }^{43} \mathrm{Ca}^{+}(\mathrm{I}=7 / 2),{ }^{87} \mathrm{Sr}^{+}(\mathrm{I}=9 / 2)$ and ${ }^{137} \mathrm{Ba}^{+}(\mathrm{I}=3 / 2)$.

\begin{tabular}{|c|c|c|c|c|c|}
\hline \multirow{5}{*}{$\begin{array}{l}\left|F, M_{F}\right\rangle \rightarrow\left|F^{\prime}, M_{F^{\prime}}\right\rangle \\
|3,0\rangle \rightarrow|2,0\rangle\end{array}$} & \multicolumn{3}{|c|}{$\overline{{ }^{43} \mathrm{Ca}^{+}}$} & \multirow{2}{*}{\multicolumn{2}{|c|}{$4 S_{1 / 2}-3 D_{5 / 2}$}} \\
\hline & \multicolumn{2}{|c|}{$4 S_{1 / 2}-3 D_{3 / 2}$} & \multirow{3}{*}{$\begin{array}{c}\left|F, M_{F}\right\rangle \rightarrow\left|F^{\prime}, M_{F^{\prime}}\right\rangle \\
|3,0\rangle \rightarrow|1,0\rangle\end{array}$} & & \\
\hline & $\lambda_{\text {magic }}$ & $\alpha_{d}^{F}\left(\lambda_{\text {magic }}\right)$ & & $\lambda_{\text {magic }}$ & $\overline{\alpha_{d}^{F}\left(\lambda_{\text {magic }}\right)}$ \\
\hline & $395.79(2)$ & 8.69 & & $395.79(3)$ & 3.35 \\
\hline & $852.5(2)$ & 95.86 & & $992(4)$ & 89.71 \\
\hline & $999(4)$ & 89.46 & $|3,0\rangle \rightarrow|2,0\rangle$ & $395.79(3)$ & 3.35 \\
\hline \multirow[t]{3}{*}{$|3,0\rangle \rightarrow|3,0\rangle$} & $395.79(2)$ & 3.35 & & $1077(9)$ & 87.31 \\
\hline & $851.3(2)$ & 95.95 & $|3,0\rangle \rightarrow|3,0\rangle$ & $395.79(3)$ & 3.35 \\
\hline & $1089(7)$ & 87.02 & & $1064(9)$ & 87.61 \\
\hline \multirow[t]{3}{*}{$|3,0\rangle \rightarrow|4,0\rangle$} & $395.79(4)$ & 3.35 & $|3,0\rangle \rightarrow|4,0\rangle$ & $395.78(3)$ & 3.35 \\
\hline & $851.7(2)$ & 95.92 & & $1032(7)$ & 88.48 \\
\hline & 1049(4) & 87.99 & $|3,0\rangle \rightarrow|5,0\rangle$ & $395.79(3)$ & 3.35 \\
\hline \multirow{3}{*}{$|3,0\rangle \rightarrow|5,0\rangle$} & $395.79(2)$ & 8.69 & & $992(4)$ & 89.71 \\
\hline & $853.4(2)$ & 95.82 & $|4,0\rangle \rightarrow|6,0\rangle$ & $395.79(3)$ & 8.69 \\
\hline & $970(3)$ & 90.44 & & $949(3)$ & 91.21 \\
\hline \multicolumn{6}{|c|}{${ }^{87} \mathrm{Sr}^{+}$} \\
\hline \multicolumn{4}{|c|}{$5 S_{1 / 2}-4 D_{3 / 2}$} & \multicolumn{2}{|c|}{$5 S_{1 / 2}-4 D_{5 / 2}$} \\
\hline$\left|\mathrm{F}, M_{F}\right\rangle \rightarrow\left|F^{\prime}, M_{F^{\prime}}\right\rangle$ & $\lambda_{\text {magic }}$ & $\alpha_{d}^{F}\left(\lambda_{\text {magic }}\right)$ & $\left|\mathrm{F}, M_{F}\right\rangle \rightarrow\left|F^{\prime}, M_{F^{\prime}}\right\rangle$ & $\lambda_{\text {magic }}$ & $\alpha_{d}^{F}\left(\lambda_{\text {magic }}\right)$ \\
\hline \multirow{3}{*}{$|4,0\rangle \rightarrow|3,0\rangle$} & $416.99(8)$ & 14.79 & $|4,0\rangle \rightarrow|3,0\rangle$ & $416.99(8)$ & 13.72 \\
\hline & $1017.4(3)$ & 108.24 & $|4,0\rangle \rightarrow|4,0\rangle$ & $416.99(7)$ & 13.72 \\
\hline & $1534(30)$ & 97.96 & $|4,0\rangle \rightarrow|5,0\rangle$ & $416.99(8)$ & 14.14 \\
\hline \multirow{2}{*}{$|4,0\rangle \rightarrow|4,0\rangle$} & $416.99(8)$ & 13.87 & $|4,0\rangle \rightarrow|6,0\rangle$ & $416.99(8)$ & 14.56 \\
\hline & $1010.7(5)$ & 108.52 & & $1486(32)$ & 98.45 \\
\hline \multirow[t]{2}{*}{$|4,0\rangle \rightarrow|5,0\rangle$} & $416.99(7)$ & 14.15 & $|5,0\rangle \rightarrow|7,0\rangle$ & $416(3)$ & 14.56 \\
\hline & $1012.4(6)$ & 108.45 & & $1301(14)$ & 100.88 \\
\hline \multirow[t]{3}{*}{$|4,0\rangle \rightarrow|6,0\rangle$} & $416.99(7)$ & 14.99 & & & \\
\hline & $1020.6(2)$ & 108.12 & & & \\
\hline & $1429(20)$ & 99.08 & & & \\
\hline \multirow{2}{*}{\multicolumn{4}{|c|}{${ }^{137} \mathrm{Ba}^{+}$}} & \multirow{2}{*}{\multicolumn{2}{|c|}{$6 S_{1 / 2}-5 D_{5 / 2}$}} \\
\hline & & & & & \\
\hline \multirow{4}{*}{$\begin{array}{l}\left|F, M_{F}\right\rangle \rightarrow\left|F^{\prime}, M_{F^{\prime}}\right\rangle \\
|1,0\rangle \rightarrow|0,0\rangle\end{array}$} & $\lambda_{\text {magic }}$ & $\alpha_{d}^{F}\left(\lambda_{\text {magic }}\right)$ & $\left|F, M_{F}\right\rangle \rightarrow\left|F^{\prime}, M_{F^{\prime}}\right\rangle$ & $\lambda_{\text {magic }}$ & $\alpha_{d}^{F}\left(\lambda_{\text {magic }}\right)$ \\
\hline & $480.6(7)$ & $\begin{array}{l}d .79 \\
-2.7\end{array}$ & $|1,0\rangle \rightarrow|1,0\rangle$ & $480.53(3)$ & 5.36 \\
\hline & $588.3(2)$ & 329.33 & & $641.2(5)$ & 256.97 \\
\hline & $695.7(9)$ & 219.39 & & & \\
\hline \multirow[t]{3}{*}{$|1,0\rangle \rightarrow|1,0\rangle$} & $480.7(4)$ & -9.37 & $|1,0\rangle \rightarrow|2,0\rangle$ & $480.58(4)$ & 0.91 \\
\hline & $587.3(3)$ & 331.55 & & $647.8(6)$ & 251.11 \\
\hline & $720.47(2)$ & 207.98 & & & \\
\hline \multirow[t]{3}{*}{$|1,0\rangle \rightarrow|2,0\rangle$} & $480.68(4)$ & -2.88 & $|1,0\rangle \rightarrow|3,0\rangle$ & $480.55(4)$ & 3.39 \\
\hline & $588.3(5)$ & 329.33 & & $644.1(5)$ & 254.42 \\
\hline & $695.7(5)$ & 219.40 & & & \\
\hline \multirow[t]{3}{*}{$|1,0\rangle \rightarrow|3,0\rangle$} & $480.56(4)$ & 3.55 & $|2,0\rangle \rightarrow|4,0\rangle$ & $480.56(5)$ & 7.84 \\
\hline & $589.7(4)$ & 329.62 & & $637.7(3)$ & 260.25 \\
\hline & $675.4(5)$ & 231.02 & & & \\
\hline
\end{tabular}

uncertainties in parentheses in the last column of this table [4]. As can be seen from the Supplemental Material, the DHF method gives large E1 matrix element values while the SD method brings down the values while the SDpT method slightly increases the values from the SD values. The scaled values from both the SD and SDpT methods modifies these values marginally. Thus, these values seem to be very reliable for determining the polarizabilities of the considered ions. For the final use, we recommend the SD method values and uncertainties to these values are estimated by taking the differences from the results obtained using the SDpT method. Below, we discuss polarizability results using these E1 matrix elements and magic wavelengths of the $S-D$ clock transitions of the above alkaline-earth ions.

\section{A. Static Polarizability results}

Using the E1 matrix elements given in Supplement Material, we first evaluate the static polarizabilities of the ground and $(n-1) D_{J}$ states of the ${ }^{43} \mathrm{Ca}^{+},{ }^{87} \mathrm{Sr}^{+}$ and ${ }^{137} \mathrm{Ba}^{+}$ions and compare them with the previously available experimental and theoretical results in Table [I] We give both the scalar and tensor polarizabilities of the considered ground and $(n-1) D_{J}$ states along with 
TABLE VIII: The $\lambda_{\mathrm{T}}$ values (in $\mathrm{nm}$ ) for all possible magnetic sublevels $M_{J}$ of the ${ }^{43} \mathrm{Ca}^{+},{ }^{87} \mathrm{Sr}^{+}$and ${ }^{137} \mathrm{Ba}^{+}$alkaline earthmetal ions using circularly polarized light.

\begin{tabular}{ccc|ccc|ccc}
\hline \hline \multicolumn{3}{c}{${ }^{43} \mathrm{Ca}^{+}$} & \multicolumn{3}{c}{${ }^{87} \mathrm{Sr}^{+}$} & \multicolumn{3}{c}{${ }^{137} \mathrm{Ba}^{+}$} \\
State & $M_{J}$ & $\lambda_{\mathrm{T}}$ & State & $M_{J}$ & $\lambda_{\mathrm{T}}$ & State & $M_{J}$ & $\lambda_{\mathrm{T}}$ \\
\hline $4 S_{1 / 2}$ & $1 / 2$ & 482.9 & $5 S_{1 / 2}$ & $-1 / 2$ & 479.2 & $6 S_{1 / 2}$ & $-1 / 2$ & 584.7 \\
& & & & $-1 / 2$ & 416.8 & & $-1 / 2$ & 478.3 \\
& & & & $-1 / 2$ & 442.3 & & $-1 / 2$ & 504.9 \\
& & & & $1 / 2$ & 417.2 & & $1 / 2$ & 482.9 \\
$3 D_{3 / 2}$ & $-1 / 2$ & 746.2 & $4 D_{3 / 2}$ & $-1 / 2$ & 881.5 & $5 D_{3 / 2}$ & $-1 / 2$ & 551.6 \\
& $1 / 2$ & 853.9 & & $1 / 2$ & 1024.7 & & $1 / 2$ & 424.4 \\
& $3 / 2$ & 851.2 & & $1 / 2$ & 467.6 & & $3 / 2$ & 590.0 \\
$3 D_{5 / 2}$ & $-1 / 2$ & 690.2 & $4 D_{5 / 2}$ & $-1 / 2$ & 834.5 & $5 D_{5 / 2}$ & $-1 / 2$ & 571.5 \\
& $1 / 2$ & 434.5 & & $1 / 2$ & 525.6 & & $1 / 2$ & 489.3 \\
& & & & & & & $3 / 2$ & 417.5 \\
\hline \hline
\end{tabular}

TABLE IX: The $\lambda_{\mathrm{T}}$ values (in $\mathrm{nm}$ ) for $F$ hyperfine levels $\left(M_{F}=0\right)$ of the ${ }^{43} \mathrm{Ca}^{+},{ }^{87} \mathrm{Sr}^{+}$and ${ }^{137} \mathrm{Ba}^{+}$alkaline earth-metal ions using circularly polarized light.

\begin{tabular}{lcc|ccc|ccc}
\hline \hline \multicolumn{3}{c}{${ }^{43} \mathrm{Ca}^{+}$} & \multicolumn{3}{c}{${ }^{87} \mathrm{Sr}^{+}$} & \multicolumn{3}{c}{${ }^{137} \mathrm{Ba}^{+}$} \\
State & $F$ & $\lambda_{\mathrm{T}}$ & State & $F$ & $\lambda_{\mathrm{T}}$ & State & $F$ & $\lambda_{\mathrm{T}}$ \\
\hline $4 S_{1 / 2}$ & 3 & 482.9 & $5 S_{1 / 2}$ & 4 & 417.1 & $6 S_{1 / 2}$ & 1 & 480.5 \\
$3 D_{3 / 2}$ & 2 & 505.7 & $4 D_{3 / 2}$ & 3 & 616.3 & $5 D_{3 / 2}$ & 0 & 472.3 \\
& & 853.4 & & & 1023.2 & & & 597.9 \\
& 3 & 467.3 & & 4 & 570.7 & & 1 & 456.6 \\
& & 881.6 & & & 1012.9 & & & 591.9 \\
& 4 & 480.8 & & 5 & 583.2 & & 2 & 472.4 \\
& & 852.2 & & & 1015.5 & & & 597.9 \\
& 5 & 526.4 & & 6 & 634.8 & & 3 & 491.3 \\
& & 854.5 & & & 1028.5 & & & 608.7 \\
$3 D_{5 / 2}$ & 1 & 495.8 & $4 D_{5 / 2}$ & 3 & 560.4 & $5 D_{5 / 2}$ & 1 & 552.5 \\
& 2 & 456.1 & & 4 & 557.9 & & 2 & 509.5 \\
& 3 & 460.2 & & 5 & 570.9 & & 3 & 519.2 \\
& 4 & 473.5 & & 6 & 596.8 & & 4 & 527.1 \\
5 & 495.8 & & 7 & 641.9 & & & \\
\hline \hline & 532.5 & & & & & & \\
\hline
\end{tabular}

their contributions from "Main" and "Tail" to valence $\alpha_{d, i}^{J, v}$, core-valence $\alpha_{d, i}^{J, c v}$ and core $\alpha_{d, i}^{J, c}$ contributions to our calculations in this table. These results are discussed ion-wise below.

${ }^{43} \mathbf{C a}^{+}$: As can be seen from Table I, $\alpha_{d, i}^{J}(0)$ value of $76.1(2)$ a.u. for the ground state polarizability obtained for ${ }^{43} \mathrm{Ca}^{+}$ion is in close agreement with the other theoretical calculations which are 75.28 a.u. and 75.49 a.u. by Tang et al. [19] and Mitroy et al. [21], respectively. Mitroy et al. have also given these values in the $3 D_{3 / 2}$ and $3 D_{5 / 2}$ states of $\mathrm{Ca}^{+}$. They had evaluated these polarizabilities by diagonalizing a semi-empirical Hamiltonian constructed in a large dimension single electron basis. Our estimated values agree quite well those values within the quoted error bars. In Ref. [20], ab initio calculations of these quantities are reported using a RCC method and our values are also compare reasonably well with them. The most stringent experimental value for the ${ }^{43} \mathrm{Ca}^{+}$ground state polarizability was obtained by spectral analysis in Ref. [22], our result is also in agreement with this value.

${ }^{87} \mathrm{Sr}^{+}$: Next, we compare our polarizability results for the ${ }^{87} \mathrm{Sr}^{+}$ion given in Table [. The RCC results of the $S$ and $D$ states reported by Sahoo et al. [24] are in agreement with our values. Mitroy et al. have also given these values by employing a non-relativistic method in the sum-over-states approach [23]. It can be seen from Table I that our ground state dipole polarizability is in very good agreement with their result. However, it seems inappropriate to compare their non-relativistic values for the dipole polarizabilities of the $4 D_{J}$ states with our relativistic calculations. The estimate for the ground state static polarizability of ${ }^{87} \mathrm{Sr}^{+}$by Barklem et al. [23] derived by combining their theoretical calculations with the experimental data from Ref. [25]. There is a considerable discrepancy between their result with our present value. This is mainly because of omission of the core contribution which has been included by us using the RPA method. There are no direct experimental results available for the $\mathrm{Sr}^{+}$ion dipole polarizabilities to make a comparative analysis with the theoretical values.

${ }^{137} \mathbf{B a}^{+}$: Our precise ground state polarizability calculation gives a value of $123.2(5)$ a.u. for the ${ }^{137} \mathrm{Ba}^{+}$ion, which is in good agreement with the high precision measurement achieved by a novel technique based on the resonant excitation Stark ionization spectroscopy [26]. We also expect that results of the $5 D_{J}$ states will also be of similar accuracies in this ion.

Having analyzed accuracies of the static polarizabilities satisfactorily, we now move on to present the dynamic polarizabilities in the above ions. We adopt the similar procedures for calculations of these quantities, thus anticipating similar accuracies in the dynamic polarizability values as their corresponding static values. This ascertains us to determine the $\lambda_{\text {magic }}$ values of the $n S-(n-1) D_{3 / 2}, n S-(n-1) D_{5 / 2}$ transitions and the $\lambda_{\mathrm{T}}$ values of the associated states in the alkaline earth metal ions without much qualm using these dynamic polarizabilities.

\section{B. Dynamic dipole polarizabilities at $1064 \mathrm{~nm}$}

We are discussing here on the dynamic polarizabilities at the $1064 \mathrm{~nm}$ that are very demanding for creating high-field seeking traps of the considered ions (far detuned traps), where the atoms are attracted to the intensity maxima. Recently Chen et al. 45] had carried out measurements of scalar and tensor contributions to the atomic polarizabilities in the $\mathrm{Rb}$ atom at this wavelength. In our calculation of dynamic polarizabilities in the considered ${ }^{43} \mathrm{Ca}^{+},{ }^{87} \mathrm{Sr}^{+}$and ${ }^{137} \mathrm{Ba}^{+}$ions, we have used same E1 matrix elements to determine the "Main" contributions and estimated other non-dominant contributions in the similar procedure as was done for the evaluation of the static polarizabilities. We list the contributions to 
the $n S_{1 / 2}$ and $(n-1) D_{J}$ dynamic polarizabilities at this wavelength of the above ions in Table III. The dominant contributions are listed explicitly. This table illustrates very fast convergence of the $n S_{1 / 2}$ state polarizabilities from which we find that the largest contributions are appearing from the $n P$ excited states. We also notice that contributions from the $4 D_{3 / 2}-5 P_{1 / 2}$ transition to the $4 D_{J}$ state polarizabilities in ${ }^{87} \mathrm{Sr}^{+}$increase 20 times as compared to the contribution from this transition to the $4 D_{J}$ state static polarizability. The reason for the overwhelming contribution from this particular transition is due to the proximity of the $4 D_{3 / 2}-5 P_{1 / 2}$ resonance that lies at $1091 \mathrm{~nm}$ to the laser wavelength of $1064 \mathrm{~nm}$.

\section{Magic and Tune out wavelengths for circularly polarized light}

In order to find out $\lambda_{\text {magic }}$ among the $\left(J, M_{J}\right)$ levels of the $n S_{1 / 2} \rightarrow(n-1) D_{3 / 2}$ and $n S_{1 / 2} \rightarrow(n-1) D_{5 / 2}$ transitions, we plot total dynamic dipole polarizabilities for the $n S_{1 / 2}$ and $(n-1) D_{3 / 2,5 / 2}$ states in Figs. (1), (2) and (3) for the ${ }^{43} \mathrm{Ca}^{+},{ }^{87} \mathrm{Sr}^{+}$and ${ }^{137} \mathrm{Ba}^{+}$ions respectively. The $\lambda_{\text {magic }}$ for the clock transitions are obtained by locating the crossing points between the two polarizability curves. In Tables [II, IV] V] and VI, we list the $\lambda_{\text {magic }}$ for the considered transitions along with their respective uncertainties in the parentheses. The corresponding polarizability values at $\lambda_{\text {magic }}$ are listed as well. The resonant wavelengths $\lambda_{\text {res }}$ are listed in the same table to demonstrate placement of a $\lambda_{\text {magic }}$ in between two resonant transitions. In this work, we use left-handed circularly polarized light $(A=-1)$ for all purposes considering all possible positive and negative $M_{J}$ sublevels for the ground $S_{1 / 2}$ and $D_{3 / 2,5 / 2}$ states. Note that $\lambda_{\text {magic }}$ for the right circularly polarized light of a transition for a given $M_{J}$ are equal to the $\lambda_{\text {magic }}$ for left circularly polarized light with opposite sign of $M_{J}$ [46].

We also investigate $\lambda_{\text {magic }}$ between the transitions involving the $\left|n S_{1 / 2} F, M_{F}=0\right\rangle$ and $\mid(n-$ 1) $\left.D_{3 / 2,5 / 2} F, M_{F}=0\right\rangle$ states. The ac Stark shifts of the hyperfine levels of an atomic state are calculated using the method described in Sec. II]. We choose $M_{F}=0$ sublevels in the hyperfine transitions as for this particular magnetic sublevel, the first order Zeeman shift vanishes. This is advantageous for the optical clock experiments [4]. We show the $\lambda_{\text {magic }}$ values of the $\mid n S_{1 / 2} F, M_{F}=$ $0\rangle \rightarrow\left|(n-1) D_{3 / 2,5 / 2} F, M_{F}=0\right\rangle$ transitions in Figs. (4), (5) and (6) for the ${ }^{43} \mathrm{Ca}^{+},{ }^{87} \mathrm{Sr}^{+}$and ${ }^{137} \mathrm{Ba}^{+}$ions respectively. These values are listed in TableVII and we discuss below about these results for the individual ion.

${ }^{43} \mathbf{C a}^{+}$: As evident from Fig. (11), the dynamic polarizabilities for the $4 S_{1 / 2}$ state are small except in the vicinity of the resonant $4 S_{1 / 2}-4 P_{1 / 2}$ and $4 S_{1 / 2}-4 P_{3 / 2}$ transitions around $396.847 \mathrm{~nm}$ and $393.366 \mathrm{~nm}$ respectively. Since the $3 D_{3 / 2,5 / 2}$ states have significant contributions from the resonances in the interested wavelength range, the $\lambda_{\text {magic }}$ are expected to lie in between these resonances.
We found a total of nine $\lambda_{\text {magic }}$ for all possible magnetic sublevels of the $4 S_{1 / 2}-3 D_{3 / 2}$ transition in between four resonances. The first four $\lambda_{\text {magic }}$ are located around $395 \mathrm{~nm}$ between the resonant $4 S_{1 / 2^{-}} 4 P_{1 / 2}$ and $4 S_{1 / 2^{-}}$ $4 P_{3 / 2}$ transitions for all the $M_{J}$ magnetic sublevels of the $4 D_{3 / 2}$ state. Out of these, two $\lambda_{\text {magic }}$ support bluedetuned trap, whereas the other two support red-detuned trap. The next five $\lambda_{\text {magic }}$ are identified at $850.9(2) \mathrm{nm}$, $853.1(2) \mathrm{nm}, 1467.8(4) \mathrm{nm}, 1013.4(5) \mathrm{nm}$ and $870.7(3)$ $\mathrm{nm}$, which lie in the infrared region. For some of the $M_{J}$ sublevels, the $\lambda_{\text {magic }}$ is missing. In such case, it would be imperative to consider a geometry where $\lambda_{\text {magic }}$ will be independent of the magnetic sublevels as mention in Sec. 1] and discussed elaborately in our previous work [47, 48]. In Table $\mathrm{V}$, we present the $\lambda_{\text {magic }}$ for the $4 S_{1 / 2}-3 D_{5 / 2}$ transition considering all the magnetic sublevels of the $3 D_{5 / 2}$ state. As seen, most of these $\lambda_{\text {magic }}$ in the ${ }^{43} \mathrm{Ca}^{+}$ ion support red detuned trap, indicated by small positive values of the polarizabilities for the corresponding $\lambda_{\text {magic }}$ values. Similarly, we tabulate $\lambda_{\text {magic }}$ for the $4 S$ $\left(M_{J}=-1 / 2\right)-3 D_{3 / 2,5 / 2}$ transition in table IV and VI It can be evidently seen from the table that $\lambda_{\text {magic }}$ are red shifted from the $\lambda_{\text {magic }}$ for $4 S\left(M_{J}=1 / 2\right)-3 D_{3 / 2,5 / 2}$.

Similarly in Table VII we list the $\lambda_{\text {magic }}$ values between 300-1300 nm for the $\left|4 S_{1 / 2} F, M_{F}=0\right\rangle \rightarrow \mid$ $\left.3 D_{3 / 2,5 / 2} F, M_{F}=0\right\rangle$ transitions. The $F$-dependent polarizabilities values at the respective $\lambda_{\text {magic }}$ are listed as well. For this wavelength range, total twenty four $\lambda_{\text {magic }}$ are located. Out of which, fourteen $\lambda_{\text {magic }}$ are identified in the infrared region. From this table, it can be found that for the $\left|4 S_{1 / 2} F, M_{F}=0\right\rangle \rightarrow\left|3 D_{3 / 2,5 / 2} F, M_{F}=0\right\rangle$ transitions, all $\lambda_{\text {magic }}$ support red-detuned traps.

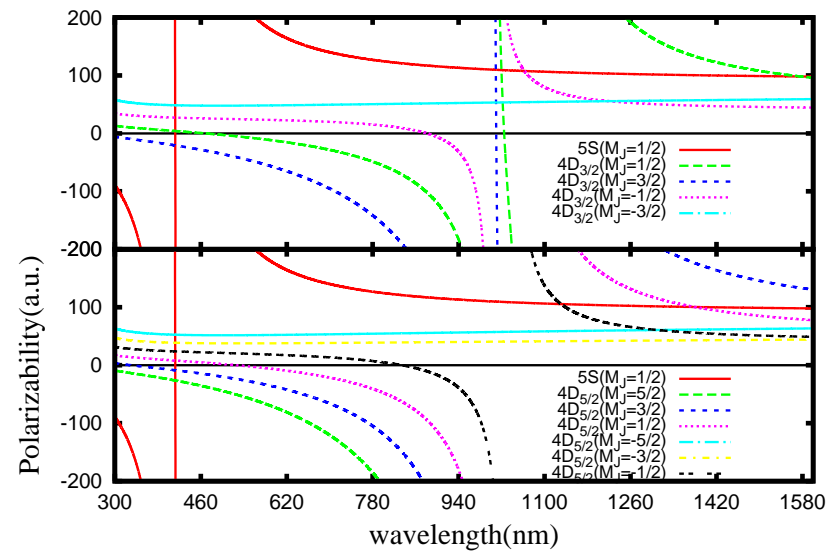

FIG. 2: (Color online) Dynamic dipole polarizabilities (in a.u.) for the $5 S_{1 / 2}$ and $4 D_{3 / 2,5 / 2}$ states of ${ }^{87} \mathrm{Sr}^{+}$with $A=-1$.

${ }^{87} \mathbf{S r}^{+}$: The dynamic polarizabilities for the $5 S_{1 / 2}$, $4 D_{3 / 2}$ and $4 D_{5 / 2}$ states of ${ }^{87} \mathrm{Sr}^{+}$calculated by us are plotted in Fig. (2). A number of $\lambda_{\text {magic }}$ are identified by the intersections of the polarizability curves of the $5 S_{1 / 2}$ and $4 D_{3 / 2,5 / 2}$ states for all their magnetic sublevels of the $4 D_{3 / 2,5 / 2}$ states in the $5 S_{1 / 2}\left(M_{J}=1 / 2\right)-4 D_{3 / 2,5 / 2}$ 
TABLE X: The $\lambda_{\text {magic }}$ values (in nm) independent of $M_{J}, F$ and $M_{F}$ with their corresponding polarizabilities (in a.u.) for the $n S_{1 / 2}-(n-1) D_{3 / 2,5 / 2}$ transition in the ${ }^{43} \mathrm{Ca}^{+},{ }^{87} \mathrm{Sr}^{+}$and ${ }^{137} \mathrm{Ba}^{+}$ions with $n=4,5,6$ respectively.

\begin{tabular}{|c|c|c|c|c|c|}
\hline \multicolumn{2}{|c|}{$\begin{array}{c}\mathrm{Ca}^{+} \\
4 S_{1 / 2}-3 D_{3 / 2} \\
\end{array}$} & \multicolumn{2}{|c|}{$\begin{array}{c}\mathrm{Sr}^{+} \\
5 S_{1 / 2}-4 D_{3 / 2} \\
\end{array}$} & \multicolumn{2}{|c|}{$\begin{array}{c}\mathrm{Ba}^{+} \\
6 S_{1 / 2}-5 D_{3 / 2}\end{array}$} \\
\hline$\lambda_{\text {magic }}$ & $\overline{\alpha_{d, 0}^{J}}\left(\lambda_{\text {magic }}\right)$ & $\lambda_{\text {magic }}$ & $\overline{\alpha_{d, 0}^{J}}\left(\lambda_{\text {magic }}\right)$ & $\lambda_{\text {magic }}$ & $\overline{\alpha_{d, 0}^{J}}\left(\lambda_{\text {magic }}\right)$ \\
\hline $395.82(3)$ & 4.90 & $416.9(3)$ & 14.47 & $480.6(5)$ & -2.89 \\
\hline $852.45(2)$ & 95.87 & $1014.6(2)$ & 108.35 & $588.4(3)$ & 329.33 \\
\hline $1029.7(2)$ & 88.55 & - & - & $655.50(3)$ & 244.89 \\
\hline \multicolumn{2}{|c|}{$4 S_{1 / 2}-3 D_{5 / 2}$} & \multicolumn{2}{|c|}{$5 S_{1 / 2}-4 D_{5 / 2}$} & \multicolumn{2}{|c|}{$6 S_{1 / 2}-5 D_{5 / 2}$} \\
\hline $395.82(2)$ & 4.20 & $416.9(3)$ & 13.3 & $480.6(4)$ & -3.75 \\
\hline $1014.10(3)$ & 89.01 & $1566.2(3)$ & 97.63 & $695.7(3)$ & 219.4 \\
\hline
\end{tabular}

TABLE XI: The $\lambda_{\mathrm{T}}$ values (in nm) independent of $M_{J}, F$ and $M_{F}$ for the $n S_{1 / 2},(n-1) D_{3 / 2}$ and $(n-1) D_{5 / 2}$ states of the ${ }^{43} \mathrm{Ca}^{+},{ }^{87} \mathrm{Sr}^{+}$and ${ }^{137} \mathrm{Ba}^{+}$ions with $n=4,5,6$ respectively.

\begin{tabular}{lc|cc|cc}
\hline \hline \multicolumn{2}{c}{${ }^{43} \mathrm{Ca}^{+}$} & \multicolumn{2}{c}{${ }^{87} \mathrm{Sr}^{+}$} & \multicolumn{2}{c}{${ }^{137} \mathrm{Ba}^{+}$} \\
State & $\lambda_{\mathrm{T}}$ & State & $\lambda_{\mathrm{T}}$ & State & $\lambda_{\mathrm{T}}$ \\
\hline $4 S_{1 / 2}$ & 395.796 & $5 S_{1 / 2}$ & 417.025 & $6 S_{1 / 2}$ & 480.596 \\
$3 D_{3 / 2}$ & 492.572 & $4 D_{3 / 2}$ & 598.633 & $5 D_{3 / 2}$ & 472.461 \\
& 852.776 & & 1018.873 & & 597.983 \\
$3 D_{5 / 2}$ & 482.642 & $4 D_{5 / 2}$ & 585.677 & $5 D_{5 / 2}$ & 509.687 \\
\hline \hline
\end{tabular}

transitions and are presented in Table III along with their resonant lines. Four $\lambda_{\text {magic }}$ are found to be around 413 nm between the $5 S_{1 / 2}-5 P_{1 / 2}$ and $5 S_{1 / 2}-5 P_{3 / 2}$, resonant transitions. These values belong to the visible region, while the other five $\lambda_{\text {magic }}$ are located at $1009.3(3) \mathrm{nm}$, $1019.7(3) \mathrm{nm}, 1062.5(3) \mathrm{nm}$ and $1577.2(3) \mathrm{nm}$ that lie in the infrared region. All the $\lambda_{\text {magic }}$ values mentioned for the $5 S_{1 / 2}-4 D_{3 / 2}$ transition, except the one at $412.5(9)$ nm, support the red-detuned trapping scheme. Similarly, for the $5 S_{1 / 2}-4 D_{5 / 2}$ transition total eight $\lambda_{\text {magic }}$ are appearing for all possible $M_{J}$ sublevels. Among them two $\lambda_{\text {magic }}$ are located at 1379.4(3) nm and 1130.8(4) nm appear after the $4 D_{5 / 2}-5 P_{3 / 2}$ resonance for the $M_{J}=1 / 2$ and $M_{J}=-1 / 2$ magnetic sublevels respectively. Use of these $\lambda_{\text {magic }}$ are recommended to carry out experiments selectively for the corresponding magnetic sublevels. Similarly, we present $\lambda_{\text {magic }}$ for the $5 S\left(M_{J}\right.$ $=-1 / 2)-4 D_{3 / 2,5 / 2}$ transition in table IV and VI It can be noticed from these tables that $\lambda_{\text {magic }}$ are red shifted from the $\lambda_{\text {magic }}$ for $5 S\left(M_{J}=1 / 2\right)-4 D_{3 / 2,5 / 2}$ transition. We have also determined total sixteen extra $\lambda_{\text {magic }}$ between $5 S_{1 / 2}-5 P_{1 / 2}$ and $4 D_{3 / 2}-5 P_{3 / 2}$ resonance transition.

In Table VII. $\lambda_{\text {magic }}$ values above $300 \mathrm{~nm}$ are listed in the case of ${ }^{87} \mathrm{Sr}^{+}$for the $\left|5 S_{1 / 2} F, M_{F}=0\right\rangle \rightarrow \mid$ $\left.4 D_{3 / 2,5 / 2} F, M_{F}=0\right\rangle$ transitions. It is found that $\lambda_{\text {magic }}$ around $417 \mathrm{~nm}$ with very small polarizabilities for the $\left|5 S_{1 / 2} F M_{F}=0\right\rangle \rightarrow\left|4 D_{3 / 2} F, M_{F}=0\right\rangle$ and $\left|5 S_{1 / 2} F M_{F}=0\right\rangle \rightarrow\left|4 D_{5 / 2} F, M_{F}=0\right\rangle$ transitions. Therefore, it will be challenging to trap the ${ }^{87} \mathrm{Sr}^{+}$ion at these wavelengths. However, the $\lambda_{\text {magic }}$ values in the infrared region for these transitions may be useful for trapping the above ion in the experiments.

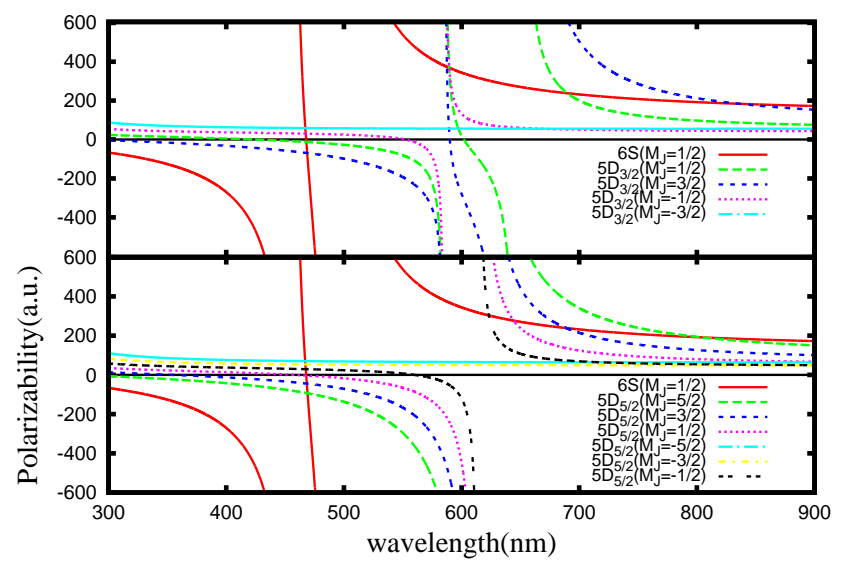

FIG. 3: (Color online) Dynamic dipole polarizabilities (in a.u.) for the $6 S_{1 / 2}$ and $5 D_{3 / 2,5 / 2}$ states of ${ }^{137} \mathrm{Ba}^{+}$with $A=-1$.

${ }^{137} \mathbf{B a}^{+}$: Total nine $\lambda_{\text {magic }}$ are found for the $6 S_{1 / 2^{-}}$ $5 D_{3 / 2}$ transition of ${ }^{137} \mathrm{Ba}^{+}$, among which four $\lambda_{\text {magic }}$ are around $468 \mathrm{~nm}$ in the vicinity of the $6 S_{1 / 2}-6 P_{3 / 2}$ resonant transition. The next $\lambda_{\text {magic }}$ at $587.6(9) \mathrm{nm}, 589.5(3)$ $\mathrm{nm}$ and 589.6(5) $\mathrm{nm}$ are located at the sharp intersection of polarizability curves close to the $5 D_{3 / 2}-6 P_{3 / 2}$ and $5 D_{3 / 2}-6 P_{1 / 2}$ resonances, as seen in Fig. (6). The last two $\lambda_{\text {magic }}$ are located at 841.7(5) $\mathrm{nm}$ and 690.7(7) $\mathrm{nm}$ for the $M_{J}=3 / 2$ and $1 / 2$ sublevel respectively, have positive polarizabilities. Hence these $\lambda_{\text {magic }}$ could provide sufficient trap depth at the reasonable laser power. In fact, some of the expected $\lambda_{\text {magic }}$ are missing for the $M_{J}=-3 / 2$ and $-1 / 2$ sublevels. Similarly, several $\lambda_{\text {magic }}$ are also located for the $6 S_{1 / 2}-5 D_{5 / 2}$ transitions, as seen from Fig. (6), in the wavelength range $300-800 \mathrm{~nm}$ which are listed in Table $\mathrm{V}$. The expected trend of locating $\lambda_{\text {magic }}$ between the resonances in this transition is similar to the previous two ions. For the $6 S\left(M_{J}=-1 / 2\right)-5 D_{3 / 2,5 / 2}$ transition, we list the $\lambda_{\text {magic }}$ in table IV and IVI These magic wavelengths are slightly red shifted to those demonstrated for $6 S\left(M_{J}=-1 / 2\right)-5 D_{3 / 2,5 / 2}$ transition. We also found total fifteen $\lambda_{\text {magic }}$ between $6 S_{1 / 2}-6 P_{1 / 2}$ and $5 D_{3 / 2}-6 P_{1 / 2}$ resonance transitions in both the tables. We have also de- 


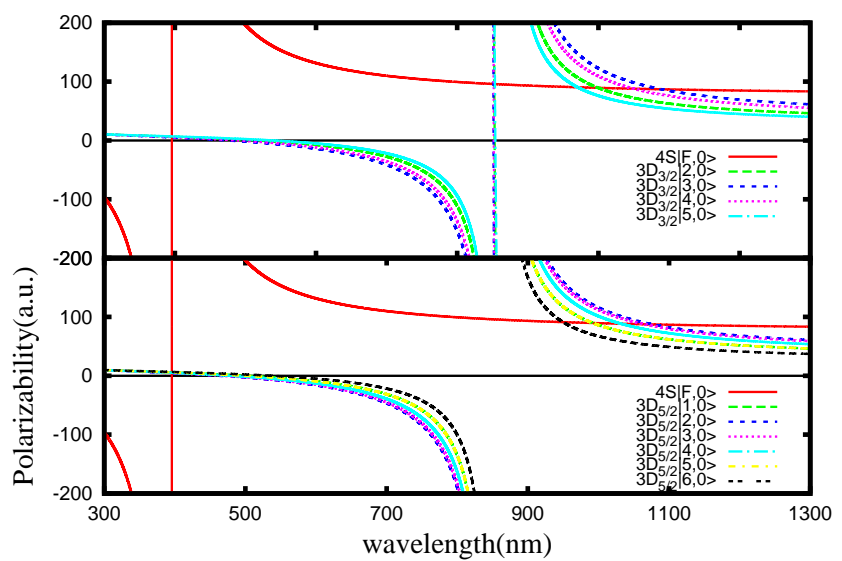

FIG. 4: (Color online) $F$ dependent dynamic dipole polarizabilities (in a.u.) for the $\left|4 S_{1 / 2} F M_{F}=0\right\rangle(F=3,4)$ and $\left|3 D_{3 / 2,5 / 2} F M_{F}=0\right\rangle$ states of ${ }^{43} \mathrm{Ca}^{+}$with $A=-1$.

termined an extra $\lambda_{\text {magic }}$ at $590.5 \mathrm{~nm}$ in table IV] which supports a red detuned trap.

In TableVII, we also list $\lambda_{\text {magic }}$ for the $\mid 6 S_{1 / 2} F, M_{F}=$ $0\rangle \rightarrow\left|5 D_{3 / 2,5 / 2} F, M_{F}=0\right\rangle$ transitions which lie within the wavelength range of $300-800 \mathrm{~nm}$. We correspondingly locate twenty $\lambda_{\text {magic }}$ in the visible region. We notice that all the $\lambda_{\text {magic }}$ values, except around $480 \mathrm{~nm}$, are expected to be more promising for experiments. An ion trap at these wavelengths can have sufficient trap depth at the reasonable laser power.

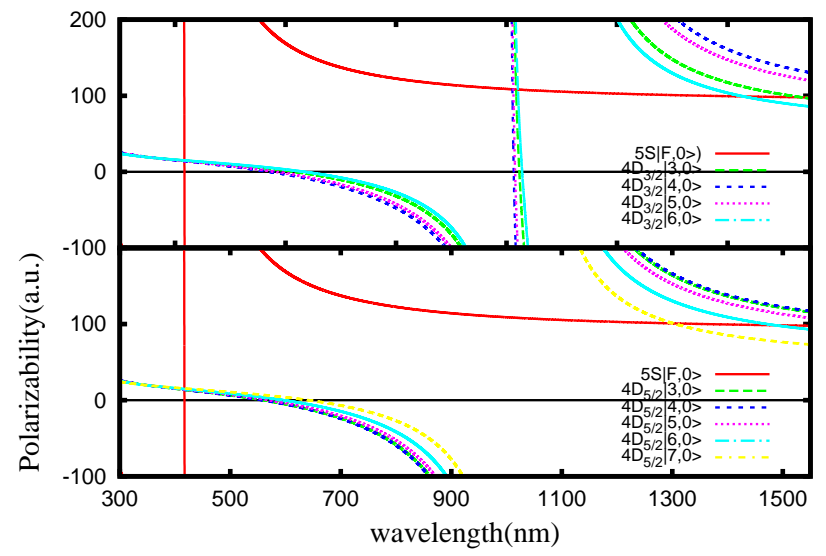

FIG. 5: (Color online) $F$ dependent dynamic dipole polarizabilities (in a.u.) for the $\left|5 S_{1 / 2} F, M_{F}=0\right\rangle(F=4,5)$ and | $\left.4 D_{3 / 2,5 / 2} F, M_{F}=0\right\rangle$ states of ${ }^{87} \mathrm{Sr}^{+}$with $A=-1$.

Tune-out wavelengths: Table VIII and IX illustrate the identified tune out wavelengths of the $n S_{1 / 2}$, $(n-1) D_{3 / 2}$ and $(n-1) D_{5 / 2}$ states in the $\left(J, M_{J}\right)$ and $\left(F, M_{F}\right)$ levels of the ${ }^{43} \mathrm{Ca}^{+},{ }^{87} \mathrm{Sr}^{+}$and ${ }^{137} \mathrm{Ba}^{+}$alkaline earth metal ions. To locate these tune out wavelengths, we have calculated the dynamic polarizabilities of the above states for a particular range of wavelength in the

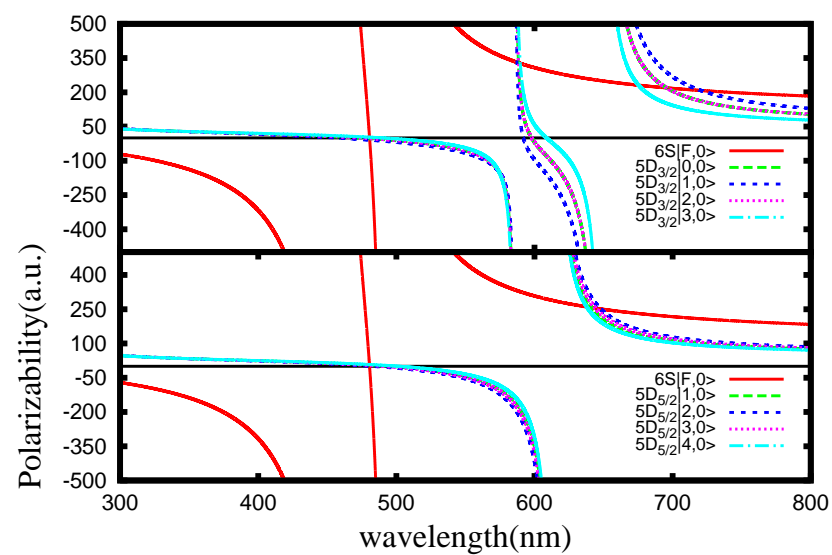

FIG. 6: (Color online) $F$ dependent dynamic dipole polarizabilities (in a.u.) for the $\left|6 S_{1 / 2} F, M_{F} 0\right\rangle(F=1,2)$ and $\left|5 D_{3 / 2,5 / 2} F, M_{F}=0\right\rangle$ states of ${ }^{137} \mathrm{Ba}^{+}$with $A=-1$.

vicinity of relevant resonances for the corresponding ion and find out values of $\lambda$ for which the polarizability values tend to zero.

\section{Magnetic sublevel independent $\lambda_{\text {magic }}$ and $\lambda_{\mathrm{T}}$}

We have also used our the frequency dependent scalar polarizability results of the ${ }^{43} \mathrm{Ca}^{+},{ }^{87} \mathrm{Sr}^{+}$and ${ }^{137} \mathrm{Ba}^{+}$ions to find out $\lambda_{\text {magic }}$ that are independent of the magnetic sublevels $M_{J}$ of the atomic states; so also hyperfine sublevel independent. Table $\mathrm{X}$ lists of the $\lambda_{\text {magic }}$ values for the $n S_{1 / 2^{-}}(n-1) D_{3 / 2,5 / 2}$ transitions, which lie within the wavelength range of 300-1500 $\mathrm{nm}$. We are also able to locate the tune out wavelengths of the ground, $(n-1) D_{3 / 2}$ and $(n-1) D_{5 / 2}$ states of the considered alkaline ions that are independent of the $F, M_{F}$ and $M_{J}$ values of the respective ion and given them in Table XI Occurrences of these $\lambda_{\text {magic }}$ and $\lambda_{\mathrm{T}}$ for the considered ions can offer pathways to carry out many high precision measurements with minimal systematics.

\section{CONCLUSION}

We have determined scalar, vector and tensor polarizabilities of the $n S_{1 / 2},(n-1) D_{3 / 2}$ and $(n-1) D_{5 / 2}$ states in the ${ }^{43} \mathrm{Ca}^{+}, \mathrm{Sr}^{+}$and ${ }^{137} \mathrm{Ba}^{+}$alkaline earth-metal ions with the ground state principal quantum number $n$. We used very precise values of the electric dipole matrix elements that are obtained them by employing a relativistic all-order method. Non-dominant contributions in the adopted sum-over-states approach for the evaluation of the polarizabilities are estimated using lower order perturbation methods. The obtained static polarizability values are compared with the available other theoretical results and experimental values to gauge their accura- 
cies. Dynamic polarizabilities at the $1064 \mathrm{~nm}$ are given explicitly for the $n S_{1 / 2}$ and $(n-1) D_{3 / 2,5 / 2}$ states of the considered alkaline earth-metal ions, which could help in creating high-field seeking traps using the Nd:YAG laser. Furthermore using the dynamic polarizabilities for a wide range of wavelengths, we have located a number of tune out wavelengths $\lambda_{\mathrm{T}}$ of the above states and the magic wavelengths $\lambda_{\text {magic }}$ for the $\left|n S_{1 / 2} F, M_{F}=0\right\rangle \rightarrow \mid$ $\left.(n-1) D_{3 / 2,5 / 2} F, M_{F}=0\right\rangle$ clock transitions due to the circularly polarized light in the ${ }^{43} \mathrm{Ca}^{+},{ }^{87} \mathrm{Sr}^{+}$and ${ }^{137} \mathrm{Ba}^{+}$ alkaline earth ions. We have located a significant number of $\lambda_{\text {magic }}$ for these clock transitions, which can help the experimentalists to trap the above ions to reduce uncertainties in the clock transitions due to Stark shifts. This knowledge would also be of immense interest to carry out other high precision studies using the considered ions. In addition, we have also determined the $\lambda_{\text {magic }}$ and $\lambda_{\mathrm{T}}$ val- ues that are independent of the choice of magnetic and hyperfine sublevels of the above clock transitions.

\section{ACKNOWLEDGEMENT}

The work of B.A. is supported by Department of Science and Technology, India and work of J. K. is supported by UGC-BSR Grant No. F.7-273/2009/BSR, India. S.S. acknowledges the financial support from UGC-BSR. A part of the computations were carried out using Vikram100 HPC cluster of Physical Research Laboratory and the employed SD method was developed in the group of Professor M. S. Safronova of the University of Delaware, USA.
[1] N. Poli, M. Schioppo, S. Vogt, S. Falke, U. Sterr, C. Lisdat, and G. M. Tino, Applied Physics B 117, 1107 (2014).

[2] T. Nicholson, S. Campbell, R. Hutson, G. Marti, B. Bloom, R. McNally, W. Zhang, M. Barrett, M. Safronova, G. Strouse, et al., Nature Communications 6, 6896 (2015).

[3] H. Katori, T. Ido, and M. K. Gonokami, J. Phys. Soc. Jpn. 68, 2479 (1999).

[4] C. Champenois, M. Houssin, C. Lisowski, M. Knoop, G. Hagel, M. Vedel, and F. Vedel, Phys. Lett. A 331, 298 (2004).

[5] C. W. Chou, D. B. Hume, J. C. J. Koelemeij, D. J. Wineland, and T. Rosenband, Phys. Rev. Lett. 104, $070802(2010)$.

[6] H. S. Margolis, G. P. Barwood, G. Huang, H. A. Klein, S. N. Lea, K. Szymaniec, and P. Gill, Science 306, 1355 (2004).

[7] E. Peik, B. Lipphardt, H. Schnatz, T. Schneider, C. Tamm, and S. G. Karshenboim, Laser Phys. 15, 1028 (2005).

[8] T. R. et al., Science 319, 1808 (2008).

[9] J. Stalnaker, S. Diddams, and T. F. et al., Appl. Phys. B 89, 167 (2007).

[10] P. L. Liu, Y. Huang, W. Bian, H. Shao, H. Guan, Y. B. Tang, C. B. Li, J. Mitroy, and K. L. Gao, Phys. Rev. Lett. 114, 223001 (2015).

[11] S. G. Karshenboim and E. Peik, Astrophysics, Clocks and Fundamental Constants, 3-540-21967-6 (Springer, Springer Verlag Berlin Heidelberg, 2004).

[12] C. A. Sackett, D. Kielpinski, B. E. King, C. Langer, V. Meyer, C. J. Myatt, M. Rowe, Q. A. Turchette, W. M. Itano, D. J. Wineland, et al., Nature 404, 256 (2000).

[13] T. Hong, C. Cramer, E. Cook, W. Nagourney, and E. N. Fortson, Opt. Lett. 30, 2644 (2005).

[14] J. Kaur, S. Singh, B. Arora, and B. K. Sahoo, Phys. Rev. A 92, 031402(R) (2015).

[15] B. K. Sahoo, R. G. E. Timmermans, B. P. Das, and D. Mukherjee, Phys. Rev. A 80, 062506 (2009).

[16] J. Sherman, W. Trimble, S. Metz, W. Nagourney, and N. Fortson, in 2005 Digest of the LEOS Summer Topical
Meetings, (IEEE), New York p. 99 (2005).

[17] B. K. Sahoo, B. P. Das, R. K. Chaudhuri, D. Mukherjee, R. G. E. Timmermans, and K. Jungmann, Phys. Rev. A 76, 040504(R) (2007).

[18] S. Burd, D. Leibfried, A. C. Wilson, and D. J. Wineland, Proc. of SPIE 9349, 93490 (2015).

[19] Y. B. Tang, H. X. Qiao, T. Y. Shi, and J. Mitroy, Phys. Rev. A 87, 042517 (2013).

[20] B. K. Sahoo, B. P. Das, and D. Mukherjee, Phys. Rev. A 79, 052511 (2009).

[21] J. Mitroy and J. Y. Zhang, Eur. Phy. J. D. 46, 415 (2008).

[22] E. S. Chang, J. Phys. B 16, 539 (1983).

[23] J. Mitroy, J. Y. Zhang, and M. W. J. Bromley, Phys. Rev. A 77, 032512 (2008).

[24] B. K. Sahoo, R. G. E. Timmermans, B. P. Das, and D. Mukherjee, Phys. Rev. A 80, 062506 (2009).

[25] P. S. Barklem and B. J. O'Mara, Mon. Not. R. Astron. Soc. 311, 535 (2000).

[26] E. L. Snow and S. R. Lundeen, Phys. Rev. A 76, 052505 (2007).

[27] S. Singh, B. K. Sahoo, and B. Arora, Phys. Rev. A 93, $063422(2016)$.

[28] M. Hobein, A. Solders, M. Suhonen, Y. Liu, and R. Schuch, Phys. Rev. Lett. 106, 013002 (2011).

[29] K. D. Bonin and V. V. Kresin, Electric-Dipole Polarizabilities of Atoms, Molecules and Clusters (World Scientific, Singapore, 1997).

[30] N. Manakov, V. Ovsiannikov, and L. Rapoport, Phys. Rep. 141, 319 (1986).

[31] K. Beloy, Ph.D. thesis (University of Nevada, 2009).

[32] V. V. Flambaum, V. A. Dzuba, and A. Derevianko, Phys. Rev. Lett. 101, 220801 (2008).

[33] B. Arora, M. S. Safronova, and C. W. Clark, Phys. Rev. A 84, 043401 (2011).

[34] L. J. LeBlanc and J. H. Thywissen, Phys. Rev. A 75, 053612 (2007).

[35] B. Arora, D. K. Nandy, and B. K. Sahoo, Phys. Rev. A 85, 012506 (2012).

[36] J. Kaur, D. K. Nandy, B. Arora, and B. K. Sahoo, Phys. Rev. A 91, 012705 (2015).

[37] A. Kramida, Y. Ralchenko, J. Reader, and N. A. 
Team, Nist atomic spectra database, ver. 5.2 (2014), http://physics.nist.gov/asd [12 Dec. 2014]. National Institute of Standards and Technology, Gaithersburg, MD.

[38] Y. Singh, B. K. Sahoo, and B. P. Das, Phys. Rev. A 88, 062504 (2013).

[39] B. K. Sahoo, L. W. Wansbeek, K. Jungmann, and R. G. E. Timmermans, Phys. Rev. A 79, 052512 (2009).

[40] S. A. Blundell, W. R. Johnson, and J. Sapirstein, Phys. Rev. A 43, 3407 (1991).

[41] W. R. Johnson, M. Idrees, and J. Sapirstein, Phys. Rev. A 35, 8 (1987).

[42] M. S. Safronova, A. Derevianko, and W. R. Johnson, Phys. Rev. A 58, 1016 (1998).
[43] M. S. Safronova and W. R. Johnson, Adv. At. Mol. Opt. Phys. 55, 191 (2007).

[44] M. S. Safronova and U. I. Safronova, Phys. Rev. A 83, 012503 (2011).

[45] Y. J. Chen, L. F. Goncalves, and G. Raithel, Phy. Rev. A 92, 060501(R) (2015).

[46] S. Singh, K. Kaur, B. K. Sahoo, and B. Arora, J. Phys. B 49, 145005 (2016).

[47] B. Arora and B. K. Sahoo, Phys. Rev. A 86, 033416 (2012).

[48] B. K. Sahoo and B. Arora, Phys. Rev. A 87, 023402 (2013). 